Document downloaded from:

http://hdl.handle.net/10251/54478

This paper must be cited as:

Vercher Martínez, A.; Giner Maravilla, E.; Arango Villegas, C.; Fuenmayor Fernández, FJ. (2015). Influence of the mineral staggering on the elastic properties of the mineralized collagen fibril in lamellar bone. Journal of the Mechanical Behavior of Biomedical Materials. 42:243-256. doi:10.1016/j.jmbbm.2014.11.022.

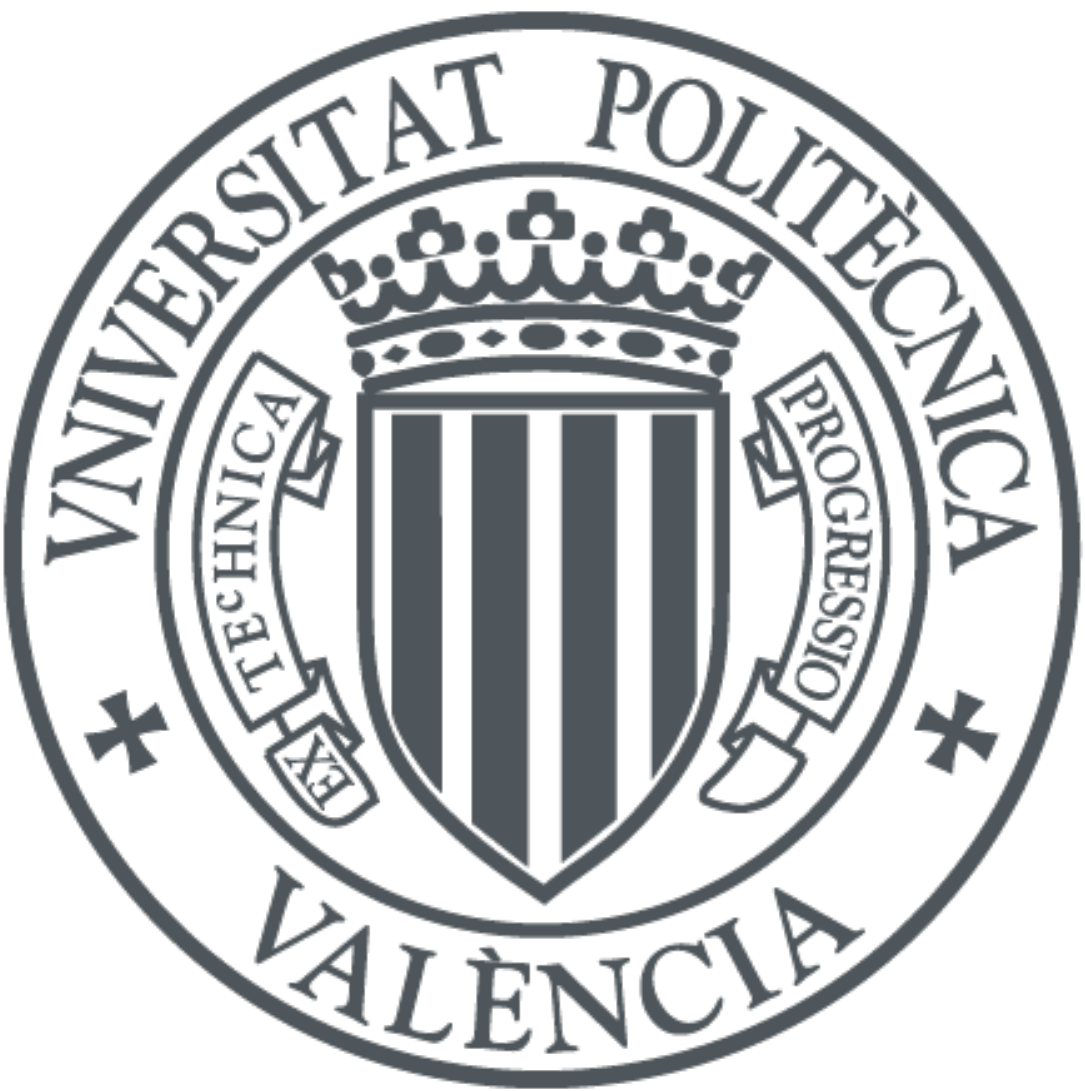

The final publication is available at

http://dx.doi.org/10.1016/j.jmbbm.2014.11.022

Copyright Elsevier

Additional Information 


\title{
Influence of the mineral staggering on the elastic properties of the mineralized collagen fibril in lamellar bone
}

\author{
Ana Vercher-Martínez*, Eugenio Giner, Camila Arango, F. Javier \\ Fuenmayor \\ Centro de Investigación en Ingeniería Mecánica - CIIM, \\ Dept. de Ingeniería Mecánica y de Materiales - CITV, \\ Universitat Politècnica de València, Camino de Vera, 46022 Valencia, Spain.
}

\begin{abstract}
In this work, a three-dimensional finite element model of the staggered distribution of the mineral within the mineralized collagen fibril has been developed to characterize the lamellar bone elastic behaviour at the sub-micro length scale. Minerals have been assumed to be embedded in a collagen matrix, and different degrees of mineralization have been considered allowing the growth of platelet-shaped minerals both in the axial and the transverse directions of the fibril, through the variation of the lateral space between platelets. We provide numerical values and trends for all the elastic constants of the mineralized collagen fibril as a function of the volume fraction of mineral. In our results, we verify the high influence of the mineral overlapping on the mechanical response of the fibril and we highlight that the lateral distance between crystals is relevant to the mechanical behaviour of the fibril and not only the mineral overlapping in the axial direction.
\end{abstract}

Keywords: Mineralized collagen fibril, lamellar bone, finite element method

${ }^{*}$ Corresponding author. Tel.: +34-96-3877007 ext. 76223; fax: +34-96-3877629.

Email address: anvermar@dimm.upv.es (Ana Vercher-Martínez) 


\section{INTRODUCTION}

Hierarchical structure of bone is a major issue to be considered in order to estimate its elastic properties (see Fig. 1). There are many works on the mechanical properties of bone by including its hierarchical structure in a multiscale model (e.g. Yoon and Cowin (2008); Hamed et al. (2010); Martínez-Reina et al. (2011); Hellmich et al. (2012)). The influence on the mechanical properties of bone, of both the constituents properties and their specific arrangement at every length scale, is nowadays an important investigation aim.

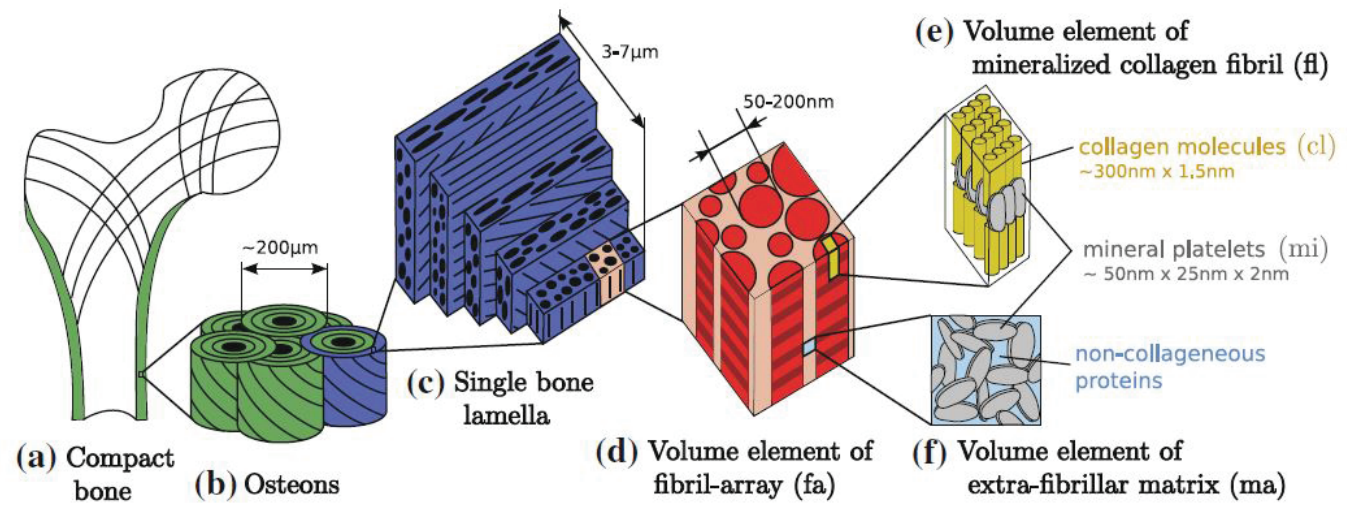

Figure 1: Hierarchical structure of cortical bone (reprinted from Reisinger et al. (2010) with permission of Springer) (a) Compact bone (b) Secondary oseons or Harverian systems (c) Single lamella of bone: composed by parallel layers (or sublayers) where fibrils rotate defining the "twisted plywood" assembly (d) Fibril-array (e) Mineralized collagen fibril, where microfibrils (not shown in the figure) can be identified by grouping five collagen molecules arranged in a staggered pattern (f) Extrafibrilar matrix

The collagen molecules in the fibril shown in Fig. 1-(e), are arranged in a quasihexagonal packing (Orgel et al., 2006) in a cross-section to the longitudinal axis of the fibril. There, the microfibrils are identified as repeating pentameric structures within the fibril (Gautier et al., 2011). A very detailed description of the collagen and mineral arrangement within the fibril is given by Silver and Landis (2012).

Molecules of collagen Type I and crystals of carbonated apatite (see Fig. 1-(e)) are the building constituents of mineralized collagen fibrils. The staggered arrangement that collagen molecules follow in the axial direction 
of the fibrils is well established in literature, e.g. Hodge and Petruska (1963); Katz and Li (1973); Weiner and Traub (1986); Landis et al. (1993); Rho et al. (1998); Orgel et al. (2001). The length of the collagen molecule is $4.4 \mathrm{D}$ (Boedtker and Doty, 1956) being $D=67 \mathrm{~nm}$ the periodic length between adjacent collagen molecules in the axial direction of the fibril. $1 D$ period is composed by the gap or hole zone of $0.6 \mathrm{D}$ and the overlap zone of $0.4 \mathrm{D}$ (Hodge and Petruska, 1963). Considering these dimensions, the total axial length of a periodic fibril model will be $5 D \approx 335 \mathrm{~nm}$ (see Figs. 2,3,4). In addition, the three dimensional assembled collagen molecules present a periodic series of gaps or channels (George and Veis, 2008). The diameter of the collagen macromolecule is about $1.23 \mathrm{~nm}$ (Landis et al., 1993) and the lateral distance between adjacent molecules is $0.24 \mathrm{~nm}$ (Lees, 1987) in absence of mineralization.

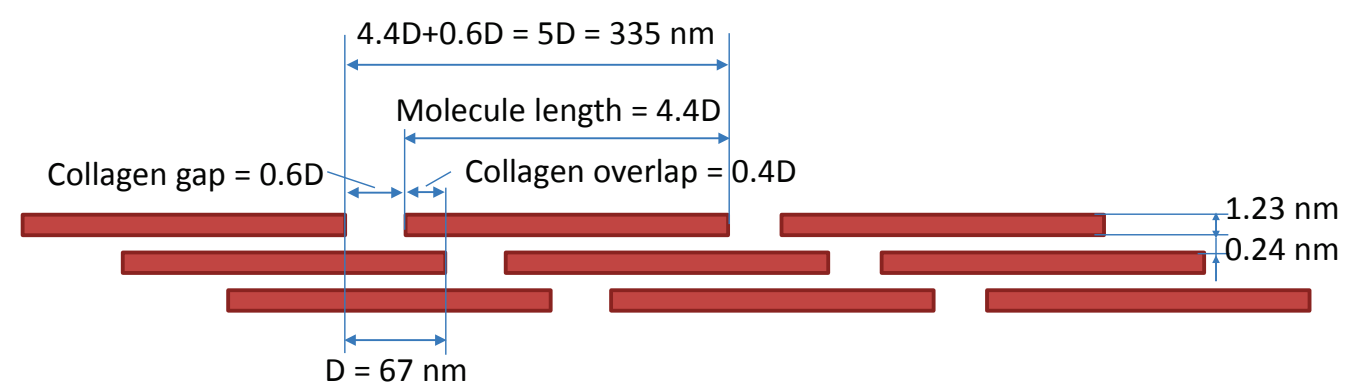

Figure 2: Schematic illustration of collagen molecule staggered arrangement inside the fibril based on Hodge and Petruska model. Diameter and lateral space between collagen molecules are from Landis et al. (1993) and Lees (1987) (not to scale)

The aggregate of collagen molecules defines the framework or scaffold where the hierarchical mineralization takes place under the control developed by basic protein functions (Liu, 2011). The periodic collagen structure will be used as a template to the mineralization within the fibril. There is evidence that, within the fibril, platelet-shaped minerals nucleate and grow in the original collagen gaps (Weiner and Traub, 1986; Fratzl et al., 1991; Silver and Landis, 2012). The platelets preferentially grow in the direction of their principal crystallographic $c$-axis and then the collagen molecule deforms (George and Veis, 2008). Platelet dimensions and their relative distance, are represented in Figs. 3,4. The platelets can be larger than the initial hole 
zone between coaxial collagen molecules, penetrating the overlap zone. In the literature, there is a wide range of values for the crystal length in the direction of the $c$-axis, $L$, e.g. we find the range $40-170 \mathrm{~nm}$ in Landis and Silver (2002) and 15-150 nm in Rubin et al. (2003). The platelet thickness, $T$, is 4-6 nm in Landis and Silver (2002) and 2-5 nm in Rubin et al. (2003). The platelet growth in the thickness direction is limited by the space between adjacent collagen molecules. The platelet width, $W$, is $30-45 \mathrm{~nm}$ in Landis and Silver (2002) and 10-80 nm in Rubin et al. (2003) and, in this direction, the crystals can coalesce to form large platelets across 3D channels formed by contiguous adjacent gaps that transverse the entire fibril (Weiner and Traub, 1986; Landis et al., 1993).

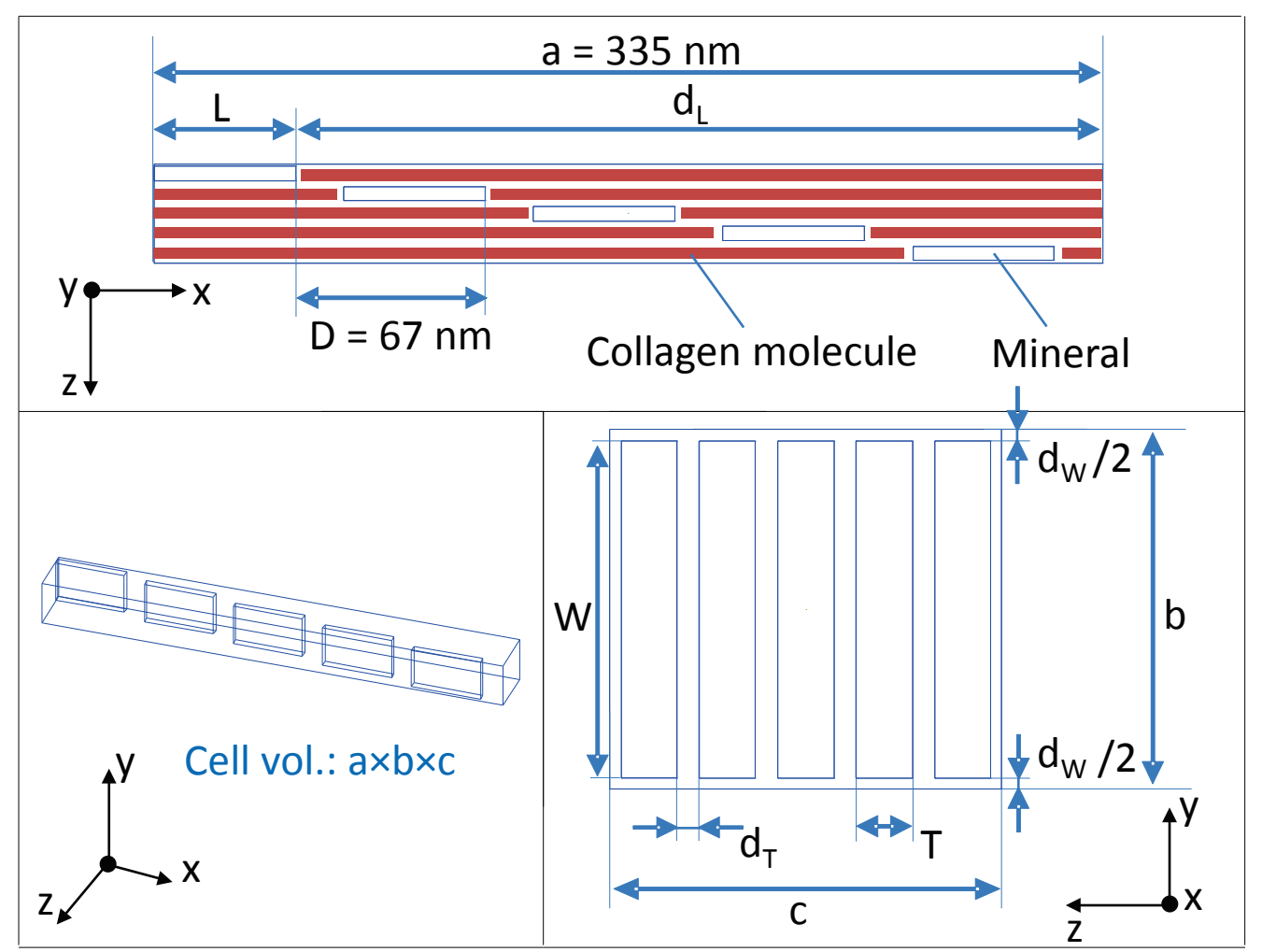

Figure 3: Unit cell volume $a \times b \times c$ and principal dimensions of the domain (in each view, proportions are preserved) 


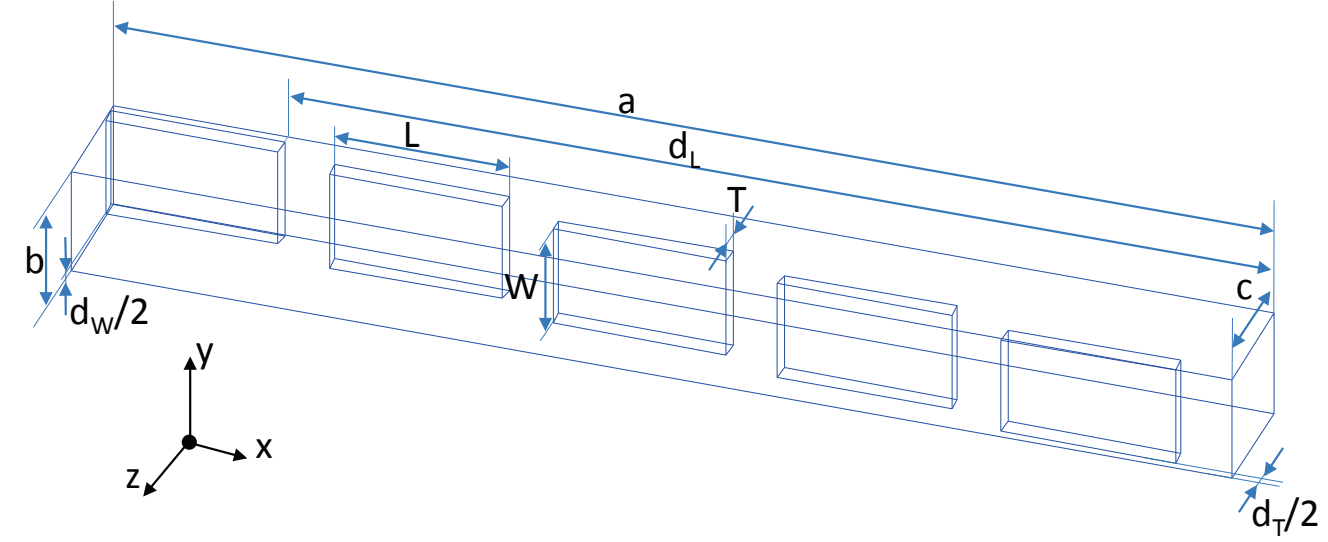

Figure 4: Three dimensional view of the unit cell volume $a \times b \times c$ and principal dimensions of the domain

Most models found in the literature consider the staggered arrangement including only overlapping in the longitudinal direction. However, after the analysis performed in this work, numerical evidence shows that it is not possible to obtain high volume fractions of mineral content (within the mineralized collagen fibrils) with only longitudinal mineral overlapping. This poses the question of what the mineral distribution should be that leads to the experimentally observed stiffness and mineral content, in addition to the mineral presence in the extra-fibrilar matrix. To avoid unrealistic platelet lengths (which should otherwise be too long), one explanation can be found in the existence of transverse mineral overlapping. In the literature, considerations about the lateral distance between neighboring crystals, $d_{T}$, are scarce. Several authors consider that this distance is of the same order than the crystal thickness (Lowenstam et al., 1989; Yuan et al., 2011; Bar-On and Wagner, 2013). On the other hand, the results shown in Fratzl et al. (1991), indicate that crystals of mineral nucleate as thin layers of calcium phosphate within the gap region of the collagen fibrils and then grow in thickness. In that work, it is observed that the collagen molecules slightly compress in the lateral space, so there could exist an overlapping between platelets in the direction of their thickness $T$, i.e. the transverse direction of the fibril. To the author's knowledge, this topic has not been numerically investigated yet. In the present work, this overlapping has been modelled using values for $d_{T} \leq 0$. 
The distance between platelets in their width direction, $d_{W}$, must be considered very small. Following Bar-On and Wagner (2013), the width of the platelets, $W$, is comparable with the width of the unit cell volume, $\mathrm{b}$, (see Fig. 3,4) because in many biological configurations the platelets are tightly packed. So, the ratio $W / b$ is usually considered almost one.

Crystal organization within the fibrils in lamellar bone is usually considered as a staggered structure of parallel layers that traverse the fibril (Katz and Li, 1973; Weiner et al., 1999; Landis et al., 1996; Erts et al., 1994; Weiner and Wagner, 1998), see Fig. 5. However, Yuan et al. (2011) consider that collagen molecules are assembled in a concentric pattern (Hulmes et al., 1995) so the platelets will grow in a concentric arrangement (Jäger and Fratzl, 2000). In recent works (Barkaoui and Hambli , 2011; Hambli and Barkaoui , 2012; Barkaoui and Hambli, 2013; Barkaoui et al. , 2014), the mechanical behaviour of bone is analyzed at the pentameric microfibril scale, by considering the collagen molecules embedded in a continuum amorphous mineral matrix.

The staggered fashion in the mineralized collagen fibril has important implications on both mechanical properties (stiffness, strength and toughness) and the mechanisms of force transmission inside the fibril (Ji and Gao, 2004). The variability and influence of the mineral shape, size and spacing between the fibril constituents, have been important investigation targets in the literature. All these parameters are closely related with the mineral content in bone whose distribution is an essential point involved in several bone diseases (Roschger et al., 2008). Jäger and Fratzl (2000) develop a 2D approach based on composite models to evaluate the influence of the distance between minerals, their thickness and volume fraction on the elastic behaviour of the fibril and how the stress is transferred depending on the distance between platelets.

In the recent work by Bar-On and Wagner (2013) an idealized 2D cell model is presented to calculate the Young's modulus in the axial direction of the fibril with analytical expressions. Halpin-Tsai equations are used for transverse Young's moduli and the results are applied to different hierarchical biological tissues. Only the influence of the mineral overlapping in the axial direction on the longitudinal Young's modulus is addressed.

In the previous work of Vercher et al. (2014), the numerical homogenization procedure by means of a finite element analysis was applied to a $4.4 \mathrm{D}$ periodic model of the staggered mineralized collagen fibril to calculate the stiffness matrix for three different volume fractions of mineral. By extending 
this procedure, and assuming that all the mineral content is concentrated within the fibril, the stiffness of lamellar bone was calculated considering the lamella definition of Weiner et al. (1999). Rotations of the mineral were taken into account by Lekhnitskii transformation.

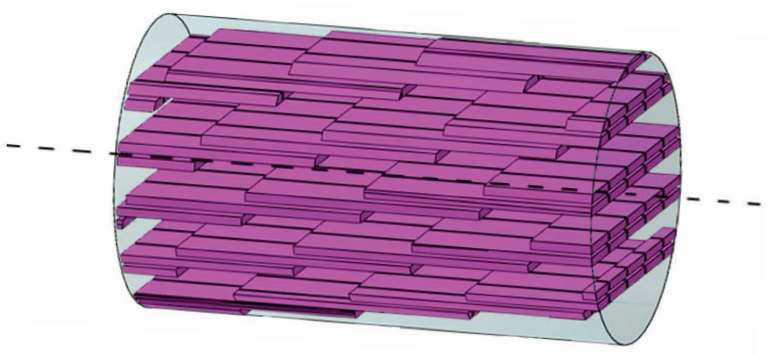

Figure 5: Hierarchical mineralization of collagen fibril: platelet distribution according to a staggered arrangement in the axial direction and parallel layers across the fibril

According to Silver and Landis (2012), a repeating unit within the fibril can be identified: five 1D-staggered collagen molecules layers. This corresponds to the cell volume analyzed in this work: a $5 \mathrm{D}(D=67 \mathrm{~nm})$ periodic model of the mineralized collagen fibril. Unlike the previous work of the authors (Vercher et al., 2014), the collagen gap zone of 0.6D has been included to calculate the length of the periodical model in the longitudinal direction of the fibril.

In this work, the hierarchical mineralization within the fibril (Liu, 2011) in lamellar bone has been modelled considering a staggered arrangement of mineral platelets in the axial direction and a distribution of parallel layers (see Fig. 5) in the transverse direction of the fibril.

A thorough study is provided to evaluate the influence of both the axial and transverse mineral overlapping on the elastic constants of the fibril. By increasing the platelet length in the preferred direction of the mineral growth, $L$, we simulate the axial overlapping. The reduction of the lateral space between platelets, $d_{T}$, leads to the overlap in the transverse direction of the fibril. The influence of the lateral overlap, reflected schematically in Fratzl et al. (1991), has been highlighted in this work. In Section 2 the numerical model and the analysis method are detailed.

In Section 3, the elastic constants of the fibrils assembly within a fibre (or fibril-array) are estimated by considering the work of Rubin et al. (2003) 
as the standpoint. In that work, the mineral rotation about its own $c$-axis is shown. To this end, the lower and upper bounds properties have been averaged.

The most relevant results are presented in Section 4 and the conclusions extracted and discussion are provided in Section 5. Finally, the Appendix contains additional results.

\section{FINITE ELEMENT ANALYSIS}

The unit cell analyzed in this work (see Figs. 3,4) aims at representing the repeating pattern in the mineralized collagen fibril: five 1D-staggered collagen molecules parallel layers, with $\mathrm{D}=67 \mathrm{~nm}$. Microfibrils are included here but are not modelled in detail. In this work, the collagen molecules in the quasihexagonal packing and, consequently, the collagen molecules in the pentameric microfibrils, are hypothesized to be a continuum matrix between the discrete mineral platelets.

A representative volume of the mineralized collagen fibril has been modelled by means of the finite element method. The numerical model considers geometric boundaries according to the structure periodicity.

In order to apply periodic boundary conditions to ensure that the hexahedron analyzed is a representative volume of the entire domain, the displacement gradients along the corresponding external surfaces must be equal. In this work, equations proposed by Hohe (2003) are used. Periodicity for the three dimensional model, implies:

$$
\begin{gathered}
u^{1+}=u^{1-}+a \epsilon_{1}^{i} \\
v^{1+}=v^{1-}+\frac{1}{2} a \epsilon_{6}^{i} \\
w^{1+}=w^{1-}+\frac{1}{2} a \epsilon_{5}^{i} \\
u^{2+}=u^{2-}+\frac{1}{2} b \epsilon_{6}^{i} \\
v^{2+}=v^{2-}+b \epsilon_{2}^{i}
\end{gathered}
$$




$$
\begin{gathered}
w^{2+}=w^{2-}+\frac{1}{2} b \epsilon_{4}^{i} \\
u^{3+}=u^{3-}+\frac{1}{2} c \epsilon_{5}^{i} \\
v^{3+}=v^{3-}+\frac{1}{2} c \epsilon_{4}^{i} \\
w^{3+}=w^{3-}+c \epsilon_{3}^{i}
\end{gathered}
$$

The above equations are applied for load cases $i=1$ to 6 (six unitary strain fields). $a, b$ and $c$ are the hexahedron dimensions (see Figs. 3,4) and the surfaces at opposite sides are indicated with symbols + and - . In addition, it is necessary to constrain the model to avoid rigid solid translations and, therefore, the central node of the finite element model is fully constrained. Rotations are implicitly constrained by the imposed periodical boundary conditions.

The fixed dimensions involved in the model are the following (see Fig. 3,4): $W=30 \mathrm{~nm}, T=5 \mathrm{~nm}, d_{W}=2 \mathrm{~nm} . d_{L}$ is calculated as $d_{L}=5 D-L \mathrm{~nm}$ and the platelet length $L$ and the lateral distance between platelets $d_{T}$ have been varied (see Figs. 6,7) inside the range proposed in literature with the aim of evaluating the influence of the mineral staggering on the elastic properties of the microfibril.

No mineral overlapping occurs in the transverse direction for positive values of $d_{T}$. The following values have been considered: 3,2 and $1 \mathrm{~nm}$, ordered by an increasing volume fraction of mineral inside the fibril. The criterion used to select these values of $d_{T}$ is that all they are of the same order than the platelet thickness (2-4.5 nm in Lowenstam et al. (1989)). For each positive value of $d_{T}, 12$ analysis have been made increasing $L$ from 40 to $150 \mathrm{~nm}$ with increments of $10 \mathrm{~nm}$. Some of the cell volumes analyzed are shown in Fig. 6.

In order to consider the mineral overlapping in the transverse direction of the fibril, the following values for $d_{T}$ have been analyzed: $0,-1,-2$, and -3 $\mathrm{nm}$. This time, for each value of $d_{T}$, only 4 cases of the platelet length have been modelled: 40, 50, 60 and $66 \mathrm{~nm}$ (no axial overlapping) in order to avoid contact between minerals (see Fig. 7).

This way, it is possible to consider a similar situation as pointed out by Fratzl et al. (1991) in their work about the analysis of the nucleation 


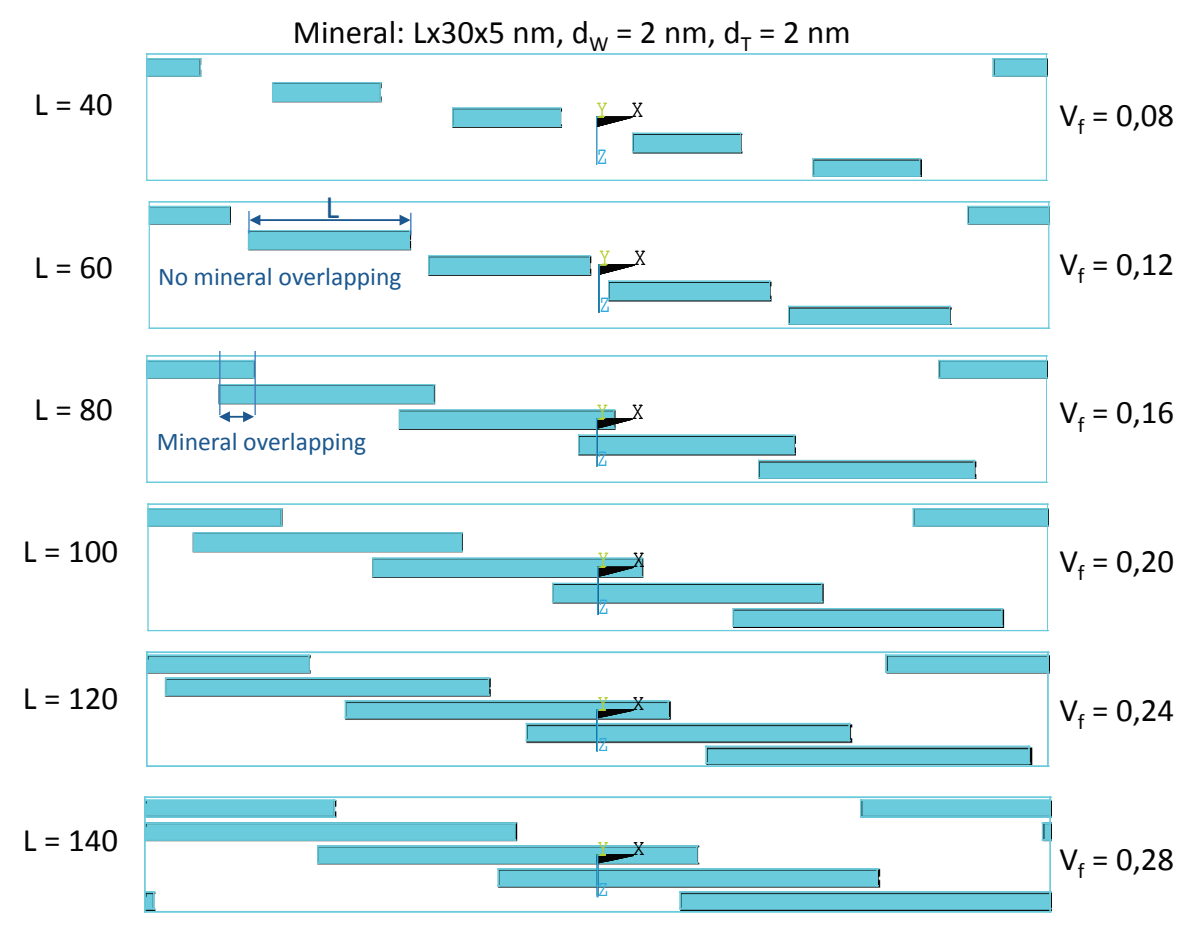

Figure 6: Example of some cell volumes analyzed with different mineral length, from $L=$ $40 \mathrm{~nm}$ to $L=140 \mathrm{~nm}$ with increments of $20 \mathrm{~nm}$. Dimensions $W, T$ and $d_{W}$ remain constant. In this figure, $d_{T}=2 \mathrm{~nm}$. The mineral is represented in blue and collagen in white

and growth of platelets. The lateral distance between collagen molecules decreases, when fibril looses their water content or when the degree of mineralization increases. For instance, when demineralized bone collagen is considered, the lateral distance between collagen molecules is $1.52 \mathrm{~nm}$ and when mineralization occurs, this distance becomes $1.24 \mathrm{~nm}$ (Bonar et al., 1985). This evidence can explains that collagen molecules can be transversally compressed when mineralization takes place.

In Fig. 8, a schematic representation of a possible arrangement that mineral crystals and collagen molecules can adopt in a portion of mineralized fibril, is shown. In this figure, all the proportions are preserved. The dotted box in Fig. 7 focuses on the approximate region of the cell volume depicted schematically in Fig. 8. A cross section in the collagen gap region, contains four collagen molecules and the distance between them. 


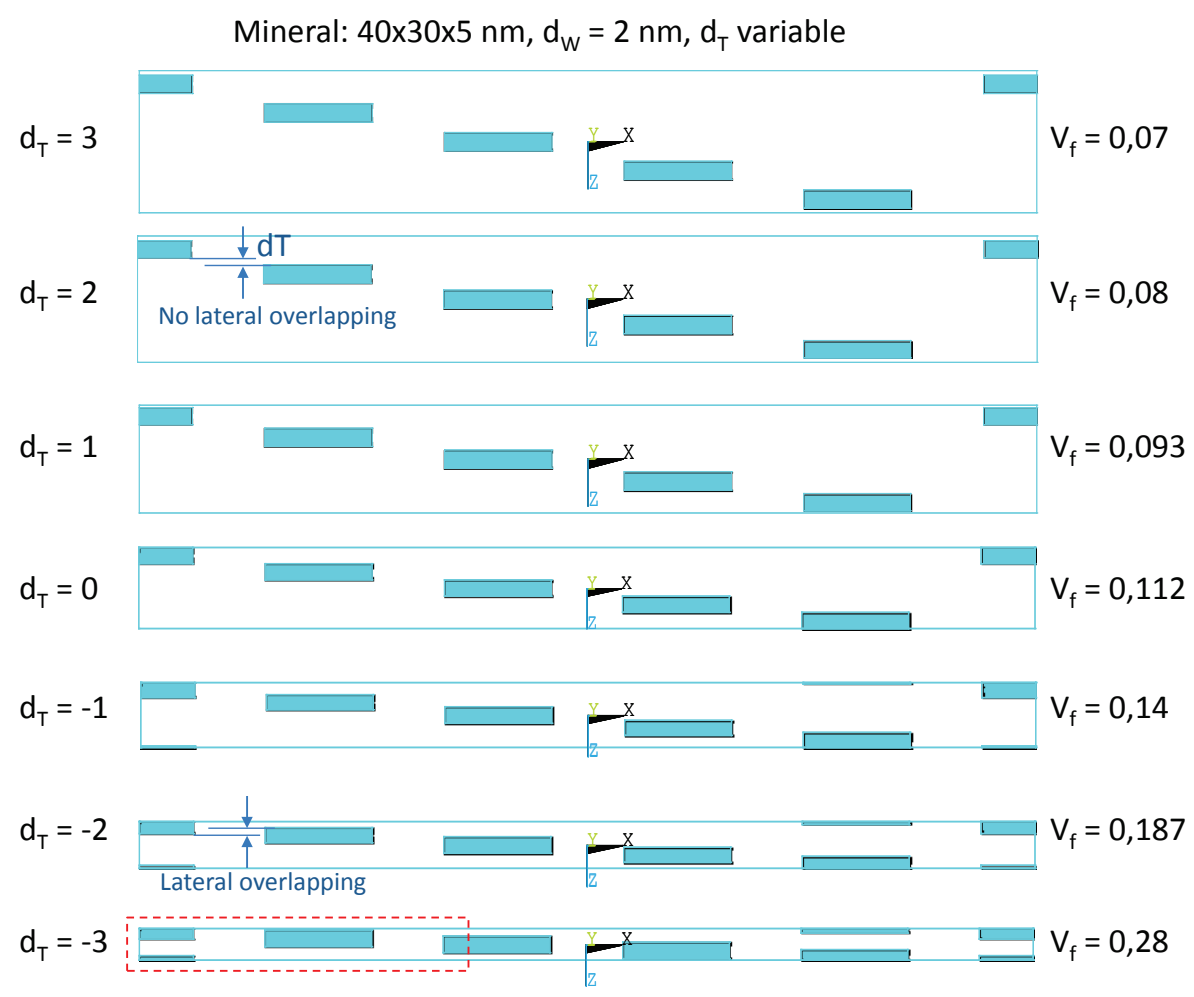

Figure 7: Example of cell volumes analyzed varying the lateral space between minerals, in the range $d_{T} \in[3,-3] \mathrm{nm}$. Dimensions $W, T$ and $d_{W}$ remain constant. In this figure, $L=40 \mathrm{~nm}$. The mineral is represented in blue and collagen in white

This scheme can be generalized for several values of $T$ and $d_{T}$. The lateral space between minerals in the section of collagen gap must be enough to host the four collagen molecules and the space between them. Therefore, the need of a minimum space implies that a certain fraction of the thickness, $T$, can be overlapped.

On the other hand, the numerical homogenization procedure described in Vercher et al. (2014) has been used to perform all the numerical analyses. The periodic boundary conditions given in Eqs. 1 to 9 with six independent unitary strain fields are applied in order to obtain the full stiffness matrix of the fibril $\mathbf{C}$.

The volume fraction of platelet reinforcement $V_{f}$ can be calculated by: 


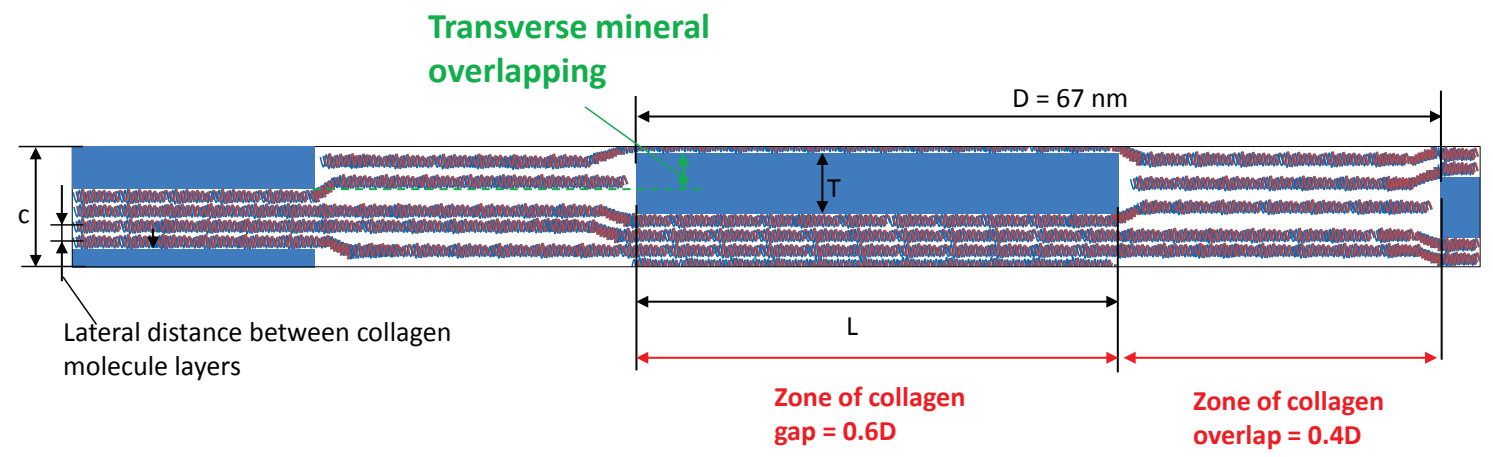

Figure 8: Schematic staggered arrangement of the collagen and mineral within the fibril with transverse overlapping of mineral. The space between adjacent parallel minerals must host four collagen molecule layers (Silver and Landis, 2012). Proportions are preserved

$$
V_{f}=\frac{L W T}{\left(L+d_{L}\right)\left(W+d_{W}\right)\left(T+d_{T}\right)}
$$

where $L$ is the platelet length, $W$ is the platelet width, $T$ is its thickness and $d_{L}, d_{W}$ and $d_{T}$ are the longitudinal, transverse in-plane and transverse outof-plane distances between platelets, respectively (see Fig. 3,4). Considering the geometrical variables of the problem whose influence has been analyzed in this work, Eq. 10 has physical meaning for $d T>-T$.

\section{FIBRIL ASSEMBLY WITHIN THE FIBRE}

In the previous section we have described the procedure followed in order to analyze the influence of both longitudinal and transverse overlapping on the stiffness of a single mineralized collagen fibril. However, it is well known that fibrils group into fibril-array at a larger scale (see Fig 1) and, within the fibrils, the minerals can rotate around its own c-axis (Akiva et al., 1998; Weiner et al., 1999; Rubin et al., 2003), see Fig. 10. Then, the spatial arrangement of the minerals in the fibril assembly is different than in a single fibril. As a consequence, the stiffness matrix of the fibril assembly will change and the mechanical behavior of the fibril-array will be different from that of a single fibril. 


\subsection{The influence of $\Psi_{2}$ angle}

In lamellar bone, the fibrils are orientated unidirectionally with a plywood angle due to the influence of the crystallographic axis of the platelet-shaped minerals (Akiva et al., 1998; Vercher et al., 2014). This orientation changes between adjacent layers of fibrils given a characteristic plywood motif of lamellar bone. The variation of the stiffness in the lamellar bone of an osteon (Franzoso and Zysset, 2009; Faingold et al., 2012) is mainly attributed to the pattern followed by the fibrils rather than the bone mineral distribution (Granke et al., 2012).
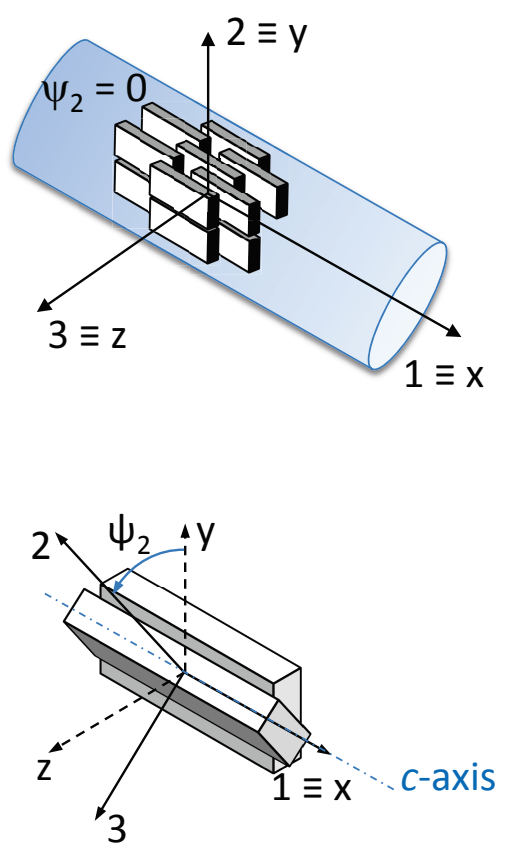

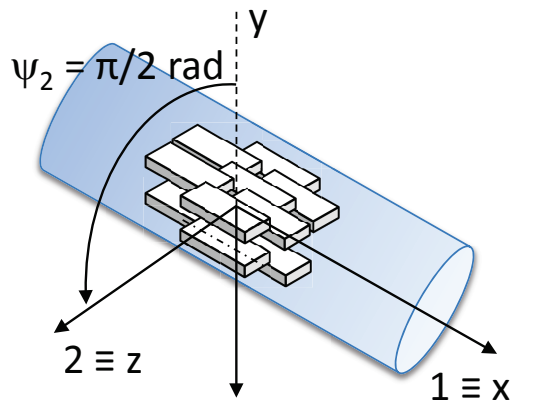

3

Figure 9: Schematic representation of the platelet rotation $\psi_{2}$ of the mineral

In addition, the mineral rotation about the $c$-axis of the crystal (angle $\Psi_{2}$ in Fig. 9) has been less thoroughly studied in the literature. In Akiva et al. (1998), $\Psi_{2}$ is constant in the fibrils that present the same unidirectional orientation. On that base, in Vercher et al. (2014) was proved that the anisotropic behaviour of the tissue is very influenced by the angle $\Psi_{2}$.

In the work by Rubin et al. (2003) a more general description is presented regarding $\Psi_{2}$. This angle does not remain constant in the set of fibrils that 
follow the same unidirectional orientation. TEM observations of Rubin show that platelets rotate around the $c$-axis in a circular pattern of $100-200 \mathrm{~nm}$ (see Fig. 10). This approach allows to expect an averaged influence of $\Psi_{2}$ for a given set of unidirectional fibrils grouped together and embedded in the extra-fibrilar matrix. From the TEM analysis of Rubin et al. (2003), it seems reasonable that the whole set of fibrils inside a fibre will present a transverse isotropic behaviour, independently of the extra-fibrilar matrix.

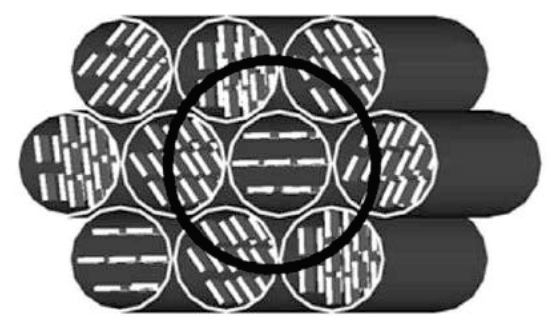

Figure 10: Schematic representation of the rotation of crystals within $200 \mathrm{~nm}$ region. Reprinted from Rubin et al. (2003) with permission of Elsevier

\subsection{Lower and upper bounds elastic properties}

The numerically homogenized stiffness matrix of the mineralized collagen fibril $\mathbf{C}$ is calculated by means of the finite element method in the coordinate system $(1,2,3)$ (see Fig 9). Then, considering the mineral rotation about the $c$-axis, the stiffness, or the compliance matrix $\mathbf{S}$, is projected in the same coordinate system $(x, y, z)$ through $\mathbf{q}^{T}\left(\Psi_{2}\right) \mathbf{C} \mathbf{q}\left(\Psi_{2}\right)$ and $\mathbf{q}^{\mathrm{T}}\left(\Psi_{2}\right) \mathbf{S} \mathbf{q}\left(\Psi_{2}\right)$ respectively, being $\mathbf{q}\left(\Psi_{2}\right)$ the Lekhnitskii transformation matrix (Lekhnitskii, 1963).

The upper and lower bounds (Hill, 1952) of the elastic properties of the fibril assembly within the fibre will be calculated by averaging the compliance matrices in the $\Psi_{2}$ range $[0,2 \pi]$ :

$$
\begin{aligned}
\mathbf{C}_{\text {Upper }} & =\frac{1}{2 \pi} \int_{0}^{2 \pi} \mathbf{q}\left(\Psi_{2}\right)^{\mathrm{T}} \mathbf{C} \mathbf{q}\left(\Psi_{2}\right) \mathrm{d} \Psi_{2} \\
\mathbf{S}_{\text {Lower }} & =\frac{1}{2 \pi} \int_{0}^{2 \pi} \mathbf{q}\left(\Psi_{2}\right)^{T} \mathbf{S} \mathbf{q}\left(\Psi_{2}\right) \mathrm{d} \Psi_{2}
\end{aligned}
$$


where $\mathbf{C}$ and $\mathbf{S}$ are the stiffness and compliance matrices, respectively, of the mineralized collagen fibril obtained from a finite element analysis and $\Psi_{2}$ is the angle that mineral rotates about its own $c$-axis depicted in Fig. 9.

It is worth noting that the integral function is calculated from 0 to $2 \pi$ because of the monoclinic behaviour of the fibril (see Vercher et al. (2014)).

The subscripts "Upper" and "Lower" refer to the corresponding upper and lower bounds of the elastic constants. By averaging the matrices $\mathbf{S}_{\text {Upper }}$ and $\mathbf{S}_{\text {Lower }}$, we define the compliance matrix $\mathbf{S}_{\text {Trans }}$, which corresponds to the behavior of the fibril assembly shown in Fig. 10. Results refer to transversely isotropic elastic properties.

\section{RESULTS}

\subsection{Influence of mineral staggering in the fibril elastic constants}

In this section, the results obtained for the analysis of Section 2 are presented. The three dimensional model has been meshed with linear solid hexahedra and the length of the largest edge is $1 \mathrm{~nm}$. In Fig. 11 the Young's moduli are represented as a function of the volume fraction of mineral that changes as a consequence of an increasing platelet length in the range $L \in$ $[40,150] \mathrm{nm}$ with increments of $10 \mathrm{~nm}$. In this case, the lateral space between platelets remains constant $d_{T}=2 \mathrm{~nm}$. Collagen and mineral have been assumed isotropic and two sets of material properties, widely used in the literature, have been considered in this analysis. The properties referenced as "Prop_1" are taken from Martínez-Reina et al. (2011): $E_{c o l}=1.2 \mathrm{GPa}$, $E_{a p}=114 \mathrm{GPa}, \nu_{c o l}=0.35$ and $\nu_{a p}=0.28$. The properties denoted as "Prop_2" are taken from Reisinger et al. (2011) and were used in the previous work of Vercher et al. (2014): $E_{c o l}=5 \mathrm{GPa}, E_{a p}=110.5 \mathrm{GPa}, \nu_{c o l}=0.3$ and $\nu_{a p}=0.28$.

Fig. 11 shows an abrupt change in the slope of $E_{x}$ and then, the curve intersects the transverse Young's modulus, $E_{y}$. This trend is also noticed in the work of Gao et al. (2003) and Bar-On and Wagner (2013,b). From the intersecting point rightwards, the mineralized collagen fibril begins to benefit from the mineral stiffness. For $d_{T}=2 \mathrm{~nm}$, this situation occurs when the platelet length is close to $67 \mathrm{~nm}$, causing the initial longitudinal mineral overlapping. However, in general, this value of the platelet length varies as a function of the $d_{T}$ distance, as we will see later. In addition, the Young's modulus $E_{z}$ is completely governed by the collagen matrix and shows, similarly to $E_{y}$, a very linear behaviour with respect to $V_{f}$. 
Note that the constituent properties have a quantitative (but not qualitative) influence on the results, principally in the results of $E_{x}$. A change in the slope of $E_{y}$ and $E_{x}$ is observed. We note in passing that this should be carefully analyzed if the materials were not isotropic. As a consequence, the remaining results of this Section are obtained considering only the set of properties named "Prop_1" and it will be hypothesized that this set of data includes the effect of the water content (a typical Young's modulus value for hydrated collagen is between $300 \mathrm{MPa}$ and $1.2 \mathrm{GPa}$, see Gautier et al. (2011)). The wet/dry properties are very important for the good characterization of the elastic constant of the fibril. In the present work, isotropic behavior and wet properties are assumed.

In Fig. 12, the Young's moduli evolution is represented as a function of the volume fraction of mineral, by varying the lateral space between platelets, $d_{T} \in[3,-3] \mathrm{nm}$ with increments of $1 \mathrm{~nm}$. For the sake of clarity, only the results for the platelet length of $L=66 \mathrm{~nm}$ are depicted. It is noticeable that again a steep change occurs in the behaviour of $E_{x}$. By reducing the lateral space between platelets, the mineralized collagen fibril can exhibit a higher stiffness in the axial direction of the fibril as a consequence of the platelet alignment. However this behaviour is also dependent on the platelet length: there exist lengths for which this phenomenon never occurs, as it will be shown later. From Figs. 11,12 it can be observed that both mineral length and lateral distance between layers have an important role in the elastic behaviour of the fibril. In this sense, it will be shown below that both variables can be closely related.

So far, only one variable has been varied each time, $L$ and $d_{T}$ respectively. In successive figures, both variables have been considered simultaneously so three dimensional graphics will be used. The surfaces result from the linear interpolation of the discrete analysis (shown with markers on the surfaces).

In Fig. 13, the same analysis as in Fig. 11 is represented but for one material only, "Prop_1". The Young's moduli variation is represented as a function of two variables: the lateral distance between adjacent platelets $d_{T}$ in the x-axis, and the platelet length $L$ in the y-axis. The dimension $d_{T}$ is varied in the range $[1,3] \mathrm{nm}$ with increments of $1 \mathrm{~nm}$. The dimension $L$ is varied in the range $[40,150] \mathrm{nm}$ with increments of $10 \mathrm{~nm}$. If we consider zero or negative values for $d_{T}$, there would be contact between platelets for values of $L$ greater than $67 \mathrm{~nm}$ and this is avoided in the analysis. The general trend is repeated again but now it is observed that the intersecting points between the surfaces $E_{x}$ and $E_{y}$ moves, meaning that when minerals 
get closer $\left(d_{T}\right.$ is reduced) the platelet length $L$ to give a stiffer fibril in the longitudinal direction is lower. As $d_{T}$ decreases, the change in the slope of $E_{x}$ is much more abrupt.

Fig. 14 shows the variation of the volume fraction of mineral $V_{f}$, with the dimensions $d_{T}$ and $L$ that take values in the same ranges than in Fig. 13. In agreement with Eq. 10, for closer minerals (lower $d_{T}$ ), the increase of $V_{f}$ with $L$ follows a greater slope.

Results shown in Fig. 15 represent the variation of the Young's moduli with the lateral distance between adjacent platelets $d_{T}$ in the x-axis, and the platelet length $L$ in the y-axis. The dimension $d_{T}$ is varied in the range $[-3,3] \mathrm{nm}$ with increments of $1 \mathrm{~nm}$ so, the mineral overlapping in the transverse direction of the fibril is considered. The dimension $L$ takes the values $L=40,50,60,66 \mathrm{~nm}$.

These results highlight that mineral overlapping in the axial direction is not strictly necessary to make the fibril stiffer in the longitudinal direction of the fibril (Bar-On and Wagner, 2013), provided that a lateral overlapping can exist. However, by reducing $d_{T}$ to negative values is not enough to make the fibril stiffer in the axial direction. In other words, a minimal platelet length is necessary.

Fig. 16 shows the variation of the volume fraction of mineral $V_{f}$ with the dimensions $d_{T}$ and $L$ that takes values in the same ranges than in Fig. 15. It is interesting to observe that the lateral space between platelets $d_{T}$, is a factor that strongly affects the mineral content in the fibrils, more than the length of platelets.

By interpolating the discrete results, the volume fraction, the platelet length and the Young's modulus $\left(E_{x}=E_{y}\right)$ corresponding to the crossing points between the surfaces $E_{x}$ and $E_{y}$ in Figs. 13,15 are summarized in Table 1.

Table 1: Values of the volume fraction of mineral $V_{f}$, platelet length $L(n m)$ and Young's modulus (GPa) for the crossing points between the surfaces $E_{x}$ and $E_{y}$ at the discrete analysis $d_{T}=-3,-2,-1,0,1,2,3(\mathrm{~nm})$

\begin{tabular}{c|c|c|c|c|c|c|c|}
$d_{T}$ & -3 & -2 & -1 & 0 & 1 & 2 & 3 \\
\hline$V_{f}$ & 0.425 & 0.284 & 0.214 & 0.175 & 0.151 & 0.137 & 0.129 \\
\hline$L$ & 60.8 & 60.9 & 61.1 & 62.7 & 64.8 & 68.4 & 73.8 \\
\hline$E_{x}=E_{y}$ & 13.47 & 9.32 & 7.31 & 6.23 & 5.49 & 5.10 & 4.88 \\
\hline
\end{tabular}


It can be noted that, as the platelets get closer laterally $\left(d_{T}<0\right)$, a lower mineral length is needed to make the fibril stiffer in the axial direction and, at the same time, the mineral content increases.

In Fig. 17, the values given on Table 1 are represented. Actually, this curve indicates the minimum platelet length for a fixed $d_{T}$ that yields a fibril stiffer in the axial direction, $x$, than in the wide transverse, $y$, where $E_{x} \geq E_{y}$. This interpretation can be done inversely, i.e. for a given platelet length $L$, the maximum lateral space in the thickness direction, $d_{T}$, is given. Any couple of points $\left(L, d_{T}\right)$ that falls within the blue shadowed area provides a mineralized collagen fibril stiffer in $x$ direction (axial) than in the $y$ direction. At the markers, the Young's moduli $\left(E_{x}=E_{y}\right.$ in GPa) and the volume fraction of mineral $V_{f}$, are indicated.

In the Appendix, the element stress components $S_{x}$ and $S_{x z}$ are represented, when a uniform positive strain is applied in the $x$ direction. The plots help to analyze the load transfer through the collagen matrix when both transverse and longitudinal mineral overlapping occurs. Additionally, results obtained for the shear moduli $G_{x y}, G_{y z}, G_{x z}$ and the major and minor Poisson ratios are summarized. The trend of the results is consistent with the behaviour observed for the Young's moduli.

\subsection{Transverse isotropic behaviour of fibril assembly within the fibre}

In this section, the results obtained for the analysis described in Section 3 are presented. The Young's moduli, shear moduli and Poisson ratios of the fibril assembly have been estimated from the transversely isotropic compliance matrix, $\mathbf{S}_{\text {trans. }}$ In Figs. 18-20 all the constants are represented as a function of the volume fraction of mineral.

The results correspond to the following dimensions and properties: $W=$ $30 \mathrm{~nm}, T=5 \mathrm{~nm}, d_{W}=2 \mathrm{~nm}, d_{T}=2 \mathrm{~nm}$. The platelet length has been varied in the range $L \in[40,150] \mathrm{nm}$ with increments of $10 \mathrm{~nm}$ considering the set of constituents properties referenced as "Prop_1".

In the $y-z$ plane, the fibril assembly exhibits an isotropic behaviour as expected. $E_{y}$ and $E_{z}$ are equal and lower than the Young's modulus in the axial direction, $E_{x}$ (see Fig.18). The anisotropic trend increases as the volume fraction of mineral does. In other works (e.g. Akkus (2005); Reisinger et al. (2010); Martínez-Reina et al. (2011)) analytical based models arrive to the same conclusion for the fibre or fibril-array. In Reisinger et al. (2011), the ratio $E_{x} / E_{y}=1.33$ calculated using nanoindetation, is provided. This ratio is an important variable to address the Young's moduli variation across 
the whole osteon (Franzoso and Zysset (2009); Faingold et al. (2012)), not only the fibril orientation pattern.

Variation of the shear moduli with the volume fraction of mineral (see Fig.19), shows a local change of behaviour as the platelet length increases. Even so, the values of $G_{x y}, G_{z x}$ and $G_{y z}$ remain quite similar in magnitude and trend, and $G_{x y}=G_{z x}$ in accordance with a transversely isotropic material. The intersecting point between curves is observed for all the lateral spaces, $d_{T}$, analyzed in this work and it always corresponds to the instant when the fibril becomes stiffer in the axial direction. Obviously, this point changes with different volume fraction of mineral. Variation of the Poisson ratios is shown in Fig.20. Results are completely compatible with the trend shown by the other elastic constants.

The results for all the elastic properties considering different $d_{T}$ have been also calculated. Results shown above prove that the monoclinic single fibril behaves mechanically in a different way when a set of them are considered following the circular pattern of the angle $\Psi_{2}$ proposed by Rubin et al. (2003).

\section{CONCLUSIONS AND DISCUSSION}

In the present work, a three-dimensional finite element model of the mineralized collagen fibril under periodic boundary conditions has been performed, to calculate the homogenized elastic properties (see Vercher et al. (2014) for details). The numerical results enable to quantify the influence of the staggered fashion on the elastic constants of the fibril by considering two geometrical variables in the model: the mineral length, $L$, and the lateral space between minerals, $d_{T}$. Both are involved in the mineral overlapping in the longitudinal and transverse directions of the fibril, respectively.

The distance $d_{T}$ is measured between the most proximal faces of the platelets in adjacent parallel layers. If transversal overlapping occurs, $d_{T}$ becomes negative. Scarce values for $d_{T}$ have been found in the literature. However, a geometrical restriction exists: there are four collagen molecule layers between successive parallel platelets (accordingly to the 5D-period model), so if we consider that the distance between collagen molecule axis in the mineralized fibril is approximately 1.25 (Fratzl et al., 1991), we need more than $3.75 \mathrm{~nm}$ to host four molecules and their lateral distances. So, the degree of compaction of the mineral, here related with $d_{T}$, is bounded by $T$ and the minimal space to contain four collagen molecules: for example, by considering the value given by Fratzl mentioned above, $d_{T}$ must be higher 
than $(3.75-4 T) / 5$. For the numerical case where $T=5 \mathrm{~nm}, d_{T}$ must be greater than $-3.25 \mathrm{~nm}$. In other words, for platelet thickness $T=5 \mathrm{~nm}$ and fully mineralized fibril, $d_{T}=-3.25 \mathrm{~nm}$ will provide the maximum degree of compaction of mineral. A higher transversal overlapping cannot occur because the four collagen molecules layers would not fit.

Certainly, to avoid the direct contact between the minerals, there are some geometrical restrictions in the model presented in this work: for values of $L$ greater or equal than $67 \mathrm{~nm}, d_{T}$ must be positive and, for values of $L$ lower than $67 \mathrm{~nm}, d_{T}$ can be either positive or negative.

This is a simplification that has been made because it is supposed that the section $L-T$ of the platelets remains in the same plane in adjacent collagen molecules layers and, if we consider a compact quasi-hexagonal packing of the collagen molecules, this is not strictly necessary and two kinds of overlapping could occur. However, this situation would seem to be contradictory with the presence of channels in the fibril if minerals are platelet-shaped. Another situation that would deal with the two kinds of overlapping simultaneously is an irregular shape of the minerals, at least at their ends. Images of this situation have been observed and can be found in Landis and Song (1991). Empirical evidence of the existence of the transversal overlapping can be found in literature (Fratzl et al., 1991; Landis and Song, 1991).

The distance between successive parallel platelets (the distance $c$ in the model (see Fig. 8) is given in literature: at least 4.2 in Landis et al. (1993). However, the values provided in the literature correspond to an initial stage of mineralization and there are no values for a fully mineralized collagen fibril, like the one supposed in our model.

Results of the first part of the work reveal that both dimensions, $L$ and $d_{T}$ are closely related in the calculus of the elastic constants of mineralized collagen fibrils. The mineral overlapping in the axial direction is not enough, and it is not the only way, to guarantee a higher stiffness of the fibril in the axial direction than in the wide transverse direction (Bar-On and Wagner, 2013). Results in this work show that as the mineral platelets get closer (low $d_{T}$ ), the mineral length that makes the fibril stiffer in the axis direction decreases, even below the necessary length to achieve the longitudinal overlapping. Explicit values for all the variables are given (see Fig. 17). A general trend that is observed is that low values of $d_{T}$ and high values of $L$ lead to a stiffer fibril (in agreement with Jäger and Fratzl (2000)).

The results of this work show that a platelet length unusually high ( $L$ greater than $100 \mathrm{~nm}$ ) is necessary to achieve typical values of volume fraction 
of mineral within the fibril (e.g. 0.238 in Martínez-Reina et al. (2011), 0.3 in Fritsch and Hellmich (2007)), if only longitudinal overlapping is considered. For these typical volume fraction, the Young's modulus of the mineralized collagen fibril in the longitudinal axis reaches values between $12-20 \mathrm{GPa}$. This range is in a good agreement with Akkus (2005), despite the collagen Young's modulus is different in this work (we compare the stiffness in the direction governed by the mineral). In turn, if transverse mineral overlapping occurs, then the Young's modulus in the axial direction is in the range $4-$ $16 \mathrm{GPa}$, for these cases the platelet length $L$ is lower than $66 \mathrm{~nm}$ (values commonly found in literature). It is noticeable that the Young's modulus $E_{y}$ is greater than $E_{x}$ for lower values of $L$ what is in contrast with MartínezReina et al. (2011). The results of the present work follow the trend given by Bar-On and Wagner (2013b) but are not directly comparable because in their work, a periodical length of $2 L$ is considered (being $L$ the platelet length) in the longitudinal direction of the fibril. In the model of this work, we assume a value of $335 \mathrm{~nm}(5 D, D=67 \mathrm{~nm})$ for this dimension, according to a five 1D-staggered collagen molecule layers (Silver and Landis, 2012).

For the Poisson ratios, there are few results in the bibliography. The results provided in this work are in agreement with the results of the analytical approach of Akkus (2005).

On the other hand, the transversely isotropic elastic constants of the fibrils assembly within a fibre have been estimated considering the observations of Rubin et al. (2003). This study provides the elastic constants of an equivalent fibril where the rotation of the minerals around the principal crystallographic $c$-axis is included.

Results in the second part of this work show that the influence of the rotation angle $\Psi_{2}$ on the elastic constants of the fibrils assembly within the fibre-array, by considering the observations of Rubin et al. (2003), is very high. In this case, a transverse isotropic behaviour is obtained (in agreement with Reisinger et al. (2010)) that tends to be more anisotropic when volume fraction of mineral increases. Following the array proposed by Rubin et al. (2003), results shown in this work reveal that the fibril assembly inside the fibre is stiffer in the axial direction regardless the presence of mineral overlapping in the individual fibrils. This allows to conclude that the influence of the angle $\Psi_{2}$ can not be neglected. In the present work, the consideration of the extra-fibrilar matrix is not addressed.

In future works it will be necessary to address the mechanisms involved in the load transmission within the fibril. To model the cross-links between 
collagen molecules is an important feature that merits a detailed study. They all play a fundamental role in load transfer. An interesting goal is to understand the failure mechanisms associated with the stress state generated for different constituents configurations in the staggered arrangement. Another important point to be considered in a future work is the influence of non isotropic wet constituents.

\section{APPENDIX: Influence of the axial mineral overlapping on shear moduli and Poisson ratios of the mineralized collagen fibril}

In order to analyze the load transfer through the collagen matrix, the element stress components $S_{x}$ and $S_{x z}$ are represented in Figs. 21,22 respectively, when a uniform positive strain is applied in the $x$ direction of the cell volume. In both figures, a constant platelet length $L=66 \mathrm{~nm}$ is considered for two different lateral space between platelets: $d_{T}=3 \mathrm{~nm}$ on the left and $d_{T}=-3 \mathrm{~nm}$ on the right. Additionally, in Figs. 23,24, the same stress components are shown under the same load case but a constant lateral space between platelets $d_{T}=1 \mathrm{~nm}$ is considered for two platelet lengths: $L=60 \mathrm{~nm}$ on the left and $L=70 \mathrm{~nm}$ on the right.

When lateral overlapping exists $\left(d_{T} \leq 0\right)$ and $L$ is sufficiently large (see Fig. 21), we have observed that the collagen matrix that is present in the regions between the crystals on the loading direction, is highly loaded in tension as a consequence of the large local strain. In the interface between collagen and the highest dimension of the mineral, $L$, the load is transferred through shear stress (see Fig. 21). Otherwise, when longitudinal overlap exists $(L>67 \mathrm{~nm})$ and $d_{T}$ is positive, but sufficiently low (see Figs. 22,24), then the collagen matrix confined in the lateral space between crystals is highly loaded in shear as a consequence of the reduction of the resistent cross area. It can be observed that the increment of the shear stress in this section produces a local bending moment near the ends of the crystals.

On the other hand, results for shear moduli and Poisson ratios are shown. For the sake of brevity, only the results corresponding to the influence of the longitudinal mineral overlapping are depicted. Different positive lateral space between minerals, $d_{T} \in[1,3] \mathrm{nm}$ have been considered, with increments of $1 \mathrm{~nm}$. For each value of $d_{T}$, the platelet length has been increased from $L=40 \mathrm{~nm}$ to $L=150 \mathrm{~nm}$ with increments of $10 \mathrm{~nm}$.

Results for shear moduli are represented in Fig. 25. The $G_{x y}$ modulus is the largest and shows a slope increment in a similar trend to $E_{x}$ (see Fig. 13). 
Both $G_{y z}$ and $G_{z x}$ show a similar behaviour and remain always lower than $G_{x y}$.

The variation of Poisson ratios with the volume fraction of mineral is shown in Figs. 26-28. These figures represent by pairs, the major and minor Poisson ratios. All the values and trends are in agreement with the results presented in Section 4, since all the values are extracted from the symmetric stiffness matrix. It can be observed that:

- The Poisson ratios $\nu_{x y}$ and $\nu_{y x}$ curves intersect following the same behaviour as $E_{x}$ and $E_{y}$.

- Since $E_{x}$ is larger than $E_{z}$, the curve of $\nu_{x z}$ remains always above $\nu_{z x}$.

- $\nu_{y z}$ is highly dominated by the collagen matrix and is higher than $\nu_{z y}$ because $E_{y}$ is greater than $E_{z}$.

- $\nu_{y x}$ and $\nu_{z x}$ present the highest reduction at a similar $V_{f}$ at which the fibril becomes stiffer in $x$ direction.

The model proposed in this work presents one plane of symmetry (plane $x-z$ ) and that is why the mineralized collagen fibril shows a monoclinic behaviour. The rest of terms (coefficients of mutual influence and Chentsov coefficients) that wholly characterize the elastic behaviour of the fibril, are quantitatively much lower than the other engineering constants.

\section{ACKNOWLEDGEMENTS}

The authors acknowledge the Ministerio de Economía y Competitividad the financial support given through the project DPI2013-46641-R and to the Programme Prometeo 2012/023.

\section{References}

Akiva, U., Wagner, H.D., Weiner, S., 1998. Modelling the three-dimensional elastic constants of parallel-fibred and lamellar bone. Journal of Materials Science 33, 1497-1509. 
Akkus, O., 2005. Elastic deformation of mineralized collagen fibrils: an equivalent inclusion based composite model. Journal of Biomechanical Engineering 127, 383-390.

Barkaoui, A., Hambli, R., 2011. Finite element 3D modeling of Mechanical Behaviour of Mineralized collagen Microfibril. Journal of Applied Biomaterials and Biomechanics 9, 207-213.

Barkaoui, A., Hambli, R., 2013. Nanomechanical properties of mineralised collagen microfibrils based on finite element method: biomechanical role of cross-links. Computer Methods in Biomechanics and Biomedical Engineering, DOI: 10.1080/10255842.2012.758255.

Barkaoui, A., Chamekh, A., Merzouki, T., Hambli, R., Mkaddem, A., 14, Multiscale approach including microfibril scale to assess elastic constants of cortical bone based on neural network computation and homogenization method. International Journal of Numerical Methods in Biomedical Engineering 30, 318-338.

Bar-On, B., Wagner, H.D., 2013. Structural motifs and elastic properties of hierarchical biological tisuues - A review. Journal of Structural Biology $183,149-164$.

Bar-On, B., Wagner, H.D., 2013. The emergence of an unusual stiffness profile in hierarchical biological tissues. Acta Biomaterialia 9, 8099-8109.

Boedtker, H., Doty, P., 1956. The native and denatured states of soluble collagen. Journal of the American Chemical Society 78, 4267-4280.

Bonar, L., Lees, S., Mook, H., 1985. Neutron diffraction studies of collagen in fully mineralized bone. Journal of Molecular Biology 181, 265-270.

Erts, D., Gathercole, L.J., Atkins, E.D.T., 1994. Scanning probe microscopy of crystallites in calcified collagen. Journal of Materials Science: Materials in Medicine 5, 200-206.

Faingold, A., Sidney, R.C., Wagner, H.D., 2012. Nanoindentation of osteonal bone lamellae. Journal of Mechanical Behavior of Biomedical Materials 9, 198-206. 
Franzoso, G., Zysset, P.K., 2009. Elastic anisotropy of human cortical bone secondary osteons measured by nanoindentation. Journal of Biomechanical Engineering 131, 021001.

Fratzl, P., Fratzl-Zelman, N., Klaushofer, K., Vogl, G., Koller, K., 1991. Nucleation and growth of mineral crystals in bone studied by small-angle x-ray scattering. Calcified Tissue International 48, 407-413.

Fritsch, A., Hellmich, C., 2007. 'Universal' microstructural pattern in cortical and trabecular, extracellular and extravascular bone materials: Micromechanics-based prediction of anisotropic elasticity. Journal of Theoretical Biology 244, 597-620.

Gao, H., Ji, B., Jager, I.L., Arzt, E., Fratzl, P., 2003. Material becomes insensitive to flaw at nanoscale: lessons from nature. Proceedings of the National Academy of Sciences 100, 5597-5600.

Gautier, A., Vesentini, S., Redaelli, A., Buehler, M.J., 2011. Hierarchical structure and nanomechanics of collagen microfibrils from the atomistic scale up. Nano Letters 11, 757-766.

George, A., Veis, A., 2008. Phosphorylated proteins and control over apatite nucleation, crystal growth and inhibition. Chemical Reviews 108, 46704693.

Granke, M., Gourrier, A., Rupin, F., Raum, K., Peyrin, F., Burghammer, M., Sad, A., Laugier, P., 2012. Microfibril orientation dominates the microeslastic properties of human bone tissue at the lamellar length scale. Plos One 8, e58043.

Gupta, H., Wagermaier, W., Zickler, G., Raz-Ben Aroush, D., Funari, S., Roschger, P., Wagner, H., Fratzl, P., 2005. Nanoscale deformation mechanisms in bone. Nano Letters 5, 2108-2111.

Hambli, R., Barkaoui, A., 2012. Physically based 3D finite element model of a single mineralized collagen microfibril. Journal of Theoretical Biology $21,28-41$.

Hamed, E., Lee, Y., Jasiuk, I., 2010. Multiscale modeling of elastic properties of cortical bone. Acta Mechanica 213, 131-154. 
Hengsberger, S., Kulik, A., Zyyset, P., 2002. Nanoindentation discriminates the elastic properties of individual human bone lamellae under dry and physiological conditions. Bone 30, 178-184.

Hellmich, Ch., Dejaco, A., Scheiner, St., 2012. Multiscale Mechanics and Mechanobiology for Bone and Bone Tissue Engineering. Journal of Tissue Engineering and Regenerative Medicine 6, 389.

Hill, R., 1952. The elastic behaviour of a crystalline aggregate. Proceedings of the Physical Society Section A 65, 349-354

Hodge, A.J., Petruska, J.A., 1963. Recent studies with the electron microscope on ordered aggregates of the tropocollagen macromolecule. In: Ramachandran, GN (Ed.), Aspects of Protein Structure. Academic Press, New York, 289-300.

Hoffler, C.E., Guo, X.E., Zysset, P.K., Goldstein, S.A., 2005. An application of nanoindentation technique to measure bone tissue lamellae properties. Journal of Biomechanical Engineering 127, 1046-1053.

Hohe, J., 2003. A direct homogenization approach for determination of the stiffness matrix for microheterogeneous plates with application to sandwich panels. Composites Part B 34, 615-626.

Hulmes, D.J.S., Wess, T.J., Prockop, D.J., Fratzl, P., 1995. Radial packing, order, and disorder in collagen fibrils. Biophysical Journal 68, 1661-1670.

Jäger, I., Fratzl, P., 2000. Mineralized collagen fibrils: a mechanical model with a staggered arrangement of mineral particles. Biophysical Journal 79, 1737-1746.

Ji, B., Gao, H., 2004. Mechanical properties of nanostructure of biological materials. Journal of the Mechanical and Physics of Solids 52, 1963-1990.

Katz, E.P., Li, S.T., 1973. The intermolecular space of reconstituted collagen fibrils. Journal of Molecular Biology 73, 351-369.

Katz, E.P., Li, S.T., 1973. Structure and function of bone collagen fibrils. Journal of Molecular Biology 80, 1-15. 
Landis, W.J., Song, M.J., 1991. Early mineral deposition in calcifying tendon characterized by high voltage electron microscopy and three-dimensional graphic imaging. Jounal of Structural Biology 107, 116-127.

Landis, W.J., Song, M.J., Leith, A., McEwen, L., McEwen, B.F., 1993. Mineral and organic matrix interaction in normally calcifying tendon visualized in three dimensions by high-voltage electron microscopic tomography and graphic image reconstruction. Journal of Structural Biology 110, 39-54.

Landis, W.J., Hodgens, K.J., Aerna, J., Song, M.J., McEwen, B.F., 1996. Structural relations between collagen and mineral in bone as determined by high voltage electron microscopic tomography. Microscopy Research and Techique 33, 192-202.

Landis, W.J., Silver, F.H., 2002. The structure and function of normally mineralizing avian tendons. Comparative Biochemestry and Physiology Part A $133,1135-1157$.

Lees, S., 1987. Considerations regarding the structure of the mammalian mineralized osteoid from viewpoint of the generalized packed model. Connective Tissue Research 16, 281-303.

Lekhnitskii, S.G., 1963. Theory of Elasticity of Anisotropic Elastic Body. Holden-Day, San Francisco, 1-73.

Liu, Y., Kim, Y.K., Dai, L., Li, N., Khan, S.O., Pashley, D.H., Tay, F.R., 2011. Hierarchical and non-hierarchical mineralization of collagen. Biomaterials $32,1291-1300$.

Lowenstam, H.A., Weiner, S., 1989. On Biomineralization. Oxford University, New York.

Martínez-Reina, J., Domínguez, J., García-Aznar, J.M., 2011. Effect of porosity and mineral content on the elastic constants of cortical bone: a multiscale approach. Biomechanics and Modeling in Mechanobiology 10, 309-322.

Orgel, J.P.R.O., Miller, A., Irving, T.C., Fischetti, R.F., Hammersley, A.P., Wess, T.J., 2001. The in situ supermolecular structure of type I collagen. Structure 9, 1061-1069. 
Orgel, J.P.R.O., Irving, T.C., Miller, A., Wess, T.J., 2006. Microfibrillar structure of type I collagn in situ. Proceedings of the National Academy of Sciences USA 103, 9001-9005.

Reisinger, A.G., Pahr, D.H., Zysset, P.K., 2010. Sensitivity analysis and parametric study of elastic properties of an unidirectional mineralized bone fibril-array using mean field methods. Biomechanics and Modeling in Mechanobiology 9, 499-510.

Reisinger, A.G., Pahr, D.H., Zysset, P.K., 2011. Elastic anisotropy of bone lamellae as a function of fibril orientation pattern. Biomechanics and Modeling in Mechanobiology 10, 67-77.

Rho, J.Y., Kuhn-Spearing, L., Zioupos, P., 1998. Mechanical properties and the hierarchical structure of bone. Medical Engineering and Physics 20, 92-102.

Roschger, P., Paschalis, E.P., Fratzl, P., Klaushofer, K., 2008. Bone mineralization density distribution in health and disease. Bone 42, 456-466.

Rubin, M.A., Jasiuk, I., Taylor, J., Rubin, J., Ganey, T., Apkarian, R.P., 2003. TEM analysis of the nanostructure of normal and osteoporotic human trabecular bone. Bone 33, 270-282.

Sasaki, N., Tagami, A., Goto, T., Taniguchi, M., Nakata, M., Hikichi, K., 2002. Atomic force microscope studies on the structure of bovine femoral cortical bone at the collagen fibril-mineral level. Journal of Materials Science: Materials in Medicine 13, 333-337.

Silver, F.H., Landis, W.J., 2012. Deposition of apatite in mineralizing vertebrate extracellular matrices: A model of possible nucleation sites on type I collagen. Connective Tissue Research 52, 242-254.

Vercher, A., Giner, E., Arango, C., Tarancón, J.E., Fuenmayor, F.J., 2014. Homogenized stiffnes matrices for mineralized collagen fibrils and lamellar bone using unit cell finite element models. Biomechanics and Modeling in Mechanobiology 13, 437-449.

Weiner, S., Traub, W., 1986. Organization of hydroxiapatite within collagen fibrils. FEBS Letters 206, 262-266. 
Weiner, S., Arad, T., Traub, W., 1991. Crystal organization in rat bone lamellae. FEBS Letters 285, 49-54.

Weiner, S., Wagner, H.D., 1998. The material bone: structure-mechanical funtion relations. Annual Review of Materials Science 28, 271-298.

Weiner, S., Traub, W., Wagner, H., 1999. Lamellar bone: structure-function relations. Journal of Structural Biology 126, 241-255.

Yoon, Y.J., Cowin, S.C., 2008b. The estimated elastic constants for a single bone osteonal lamella. Biomechanics and Modeling in Mechanobiology 7, $1-11$.

Yuan, F., Stock, S.R., Haeffner, D.R., Almer, J.D., Dunand, D.C., Brinson, L.C., 2011. A new model to simulate the elastic properties of mineralized collagen fibril. Biomechanics and Modeling in Mechanobiology 10, 147-160. 


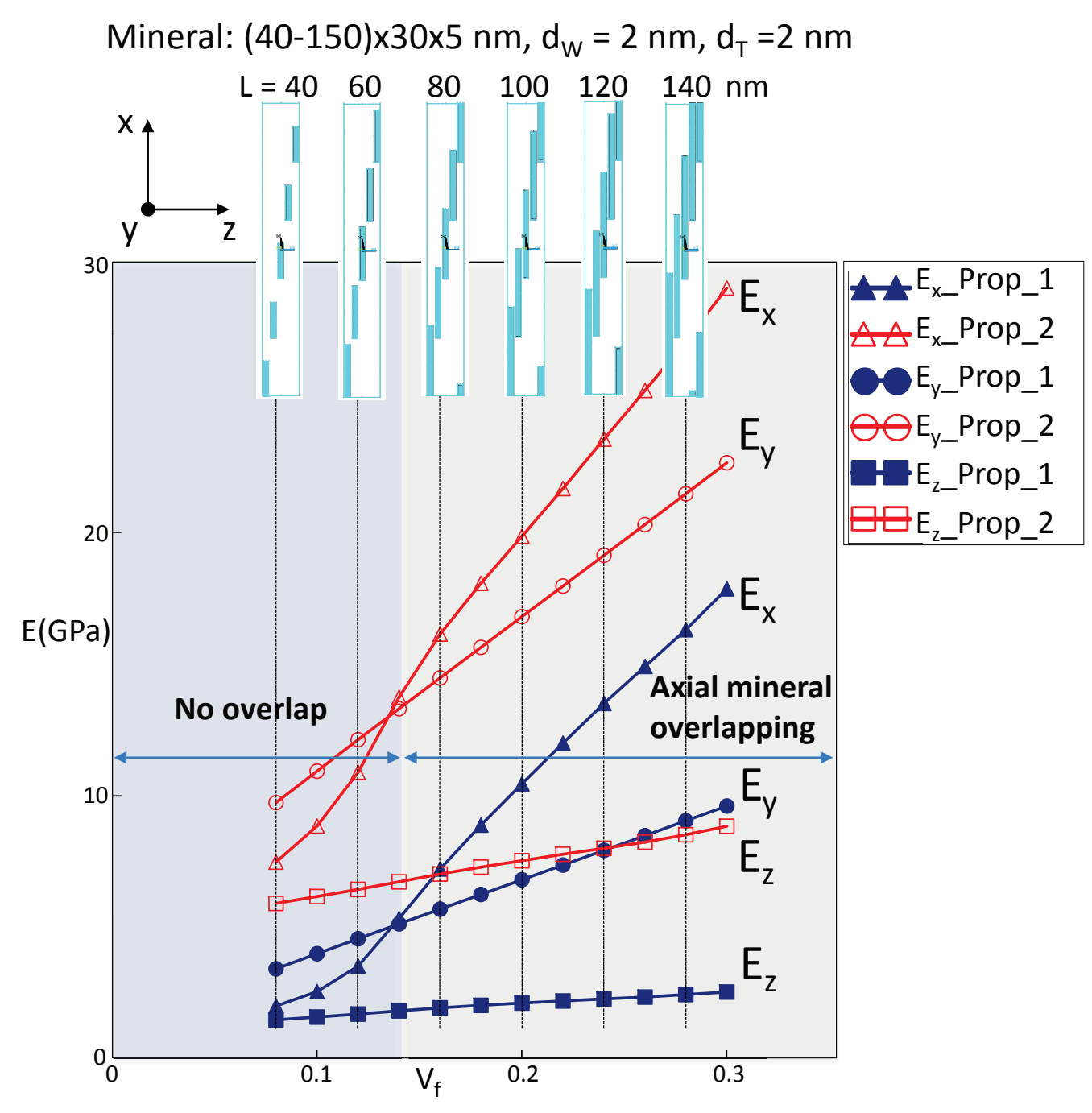

Figure 11: Variation of Young's moduli with volume fraction of mineral by considering different platelet lengths, in the range $L \in[40,150] \mathrm{nm}$. Results are obtained for two sets of constituent elastic properties. Lateral space between platelets, $d_{T}$, remains constant and equal to $2 \mathrm{~nm}$ 


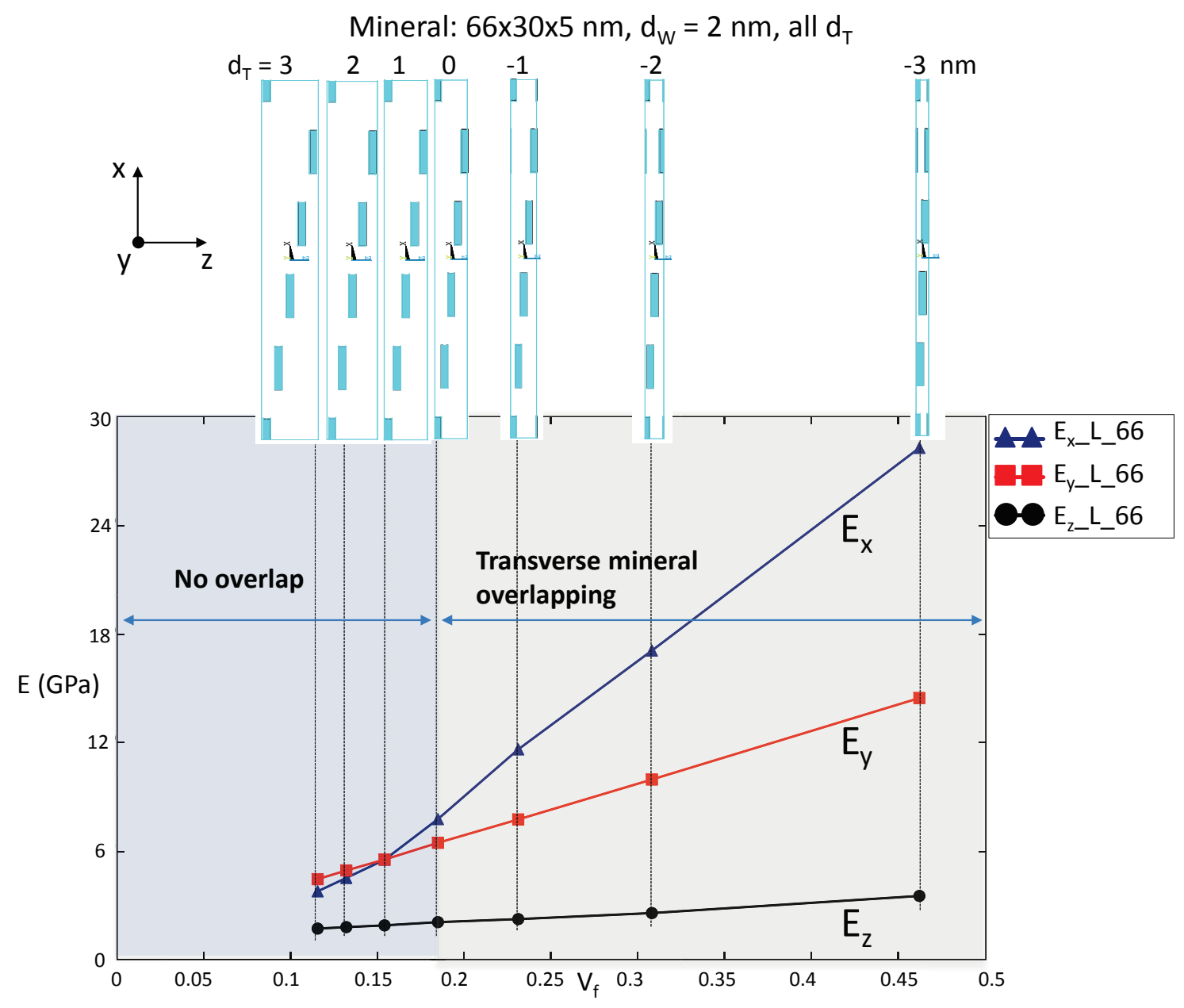

Figure 12: Variation of Young's moduli with volume fraction of mineral by considering different lateral space between platelets, in the range $d_{T} \in[3,-3] \mathrm{nm}$. Results are obtained for a constant platelet length, $L=66 \mathrm{~nm}$ 
Mineral: $(40-150) \times 30 \times 5 \mathrm{~nm}, \mathrm{~d}_{\mathrm{W}}=2 \mathrm{~nm}, \mathrm{~d}_{\mathrm{T}}>0 \mathrm{~nm}$

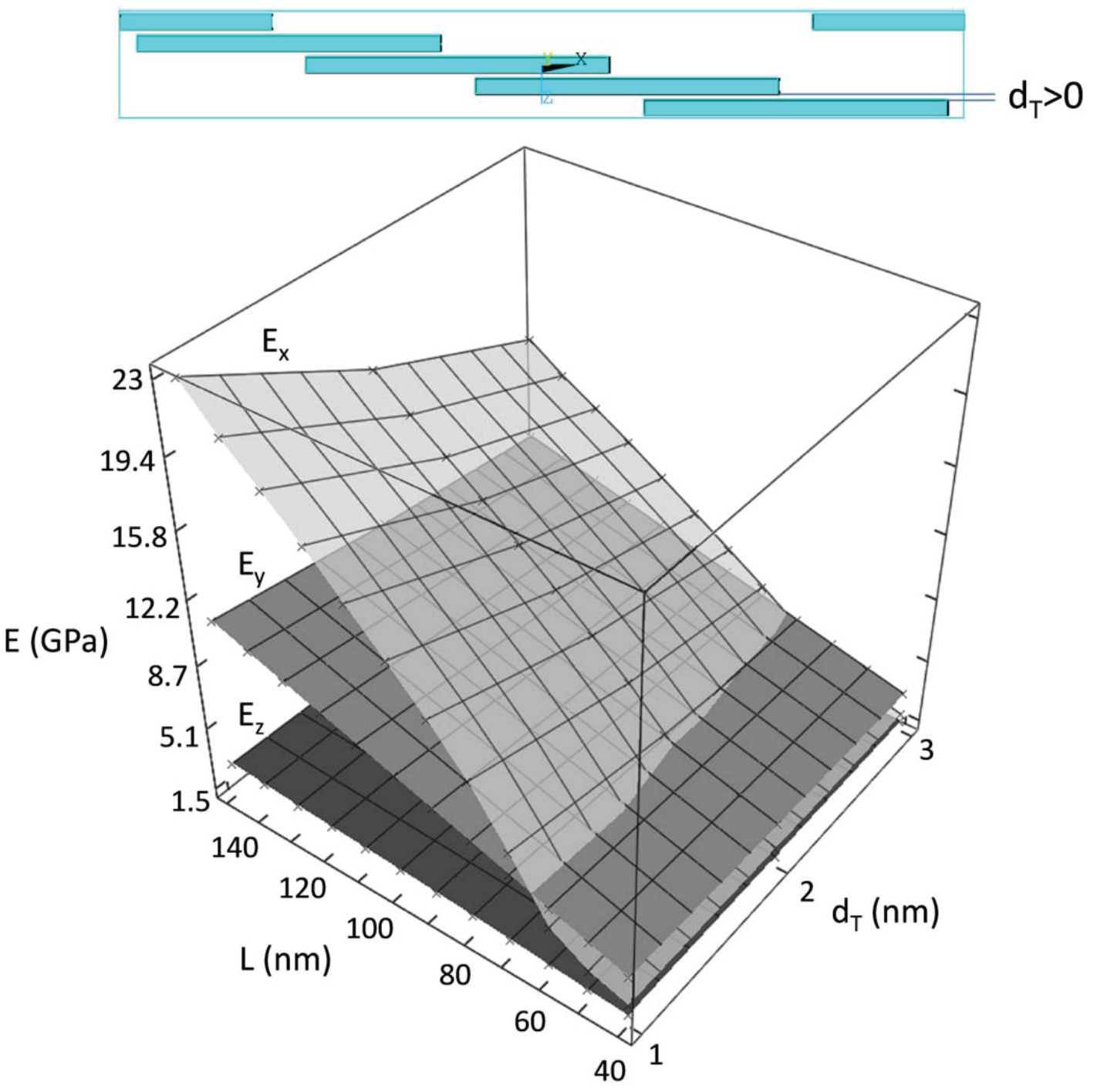

Figure 13: Variation of Young's moduli with the lateral distance $d_{T}$, in the range [1,3] nm, and the platelet length $L$, in the range $[40,150] \mathrm{nm}$ 
Mineral: $(40-150) \times 30 \times 5 \mathrm{~nm}, \mathrm{~d}_{\mathrm{W}}=2 \mathrm{~nm}, \mathrm{~d}_{\mathrm{T}}>0 \mathrm{~nm}$

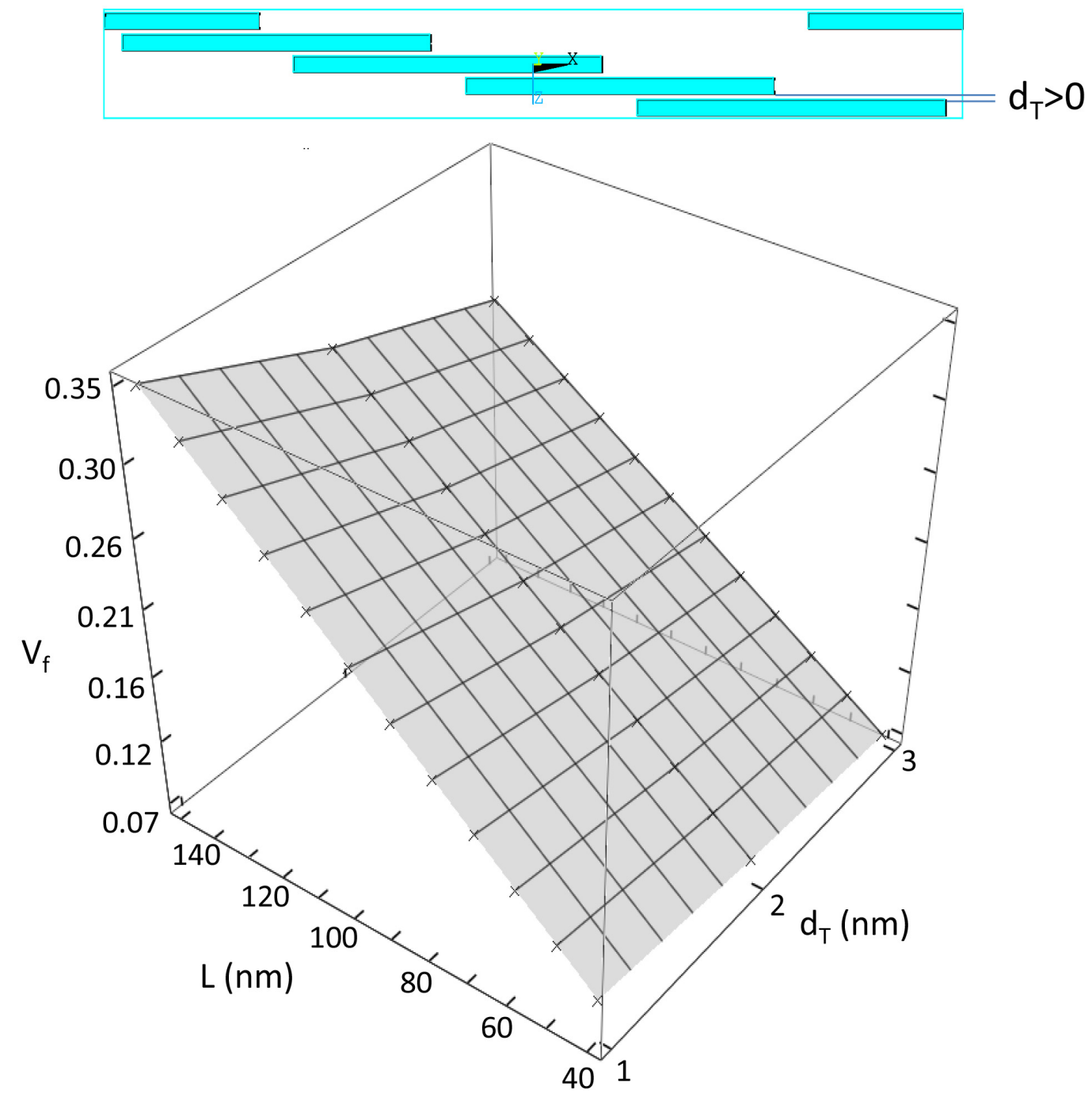

Figure 14: Variation of volume fraction of mineral $V_{f}$ with the lateral distance $d_{T}$, in the range $[1,3] \mathrm{nm}$ and the platelet length $L$, in the range $[40,150] \mathrm{nm}$ 

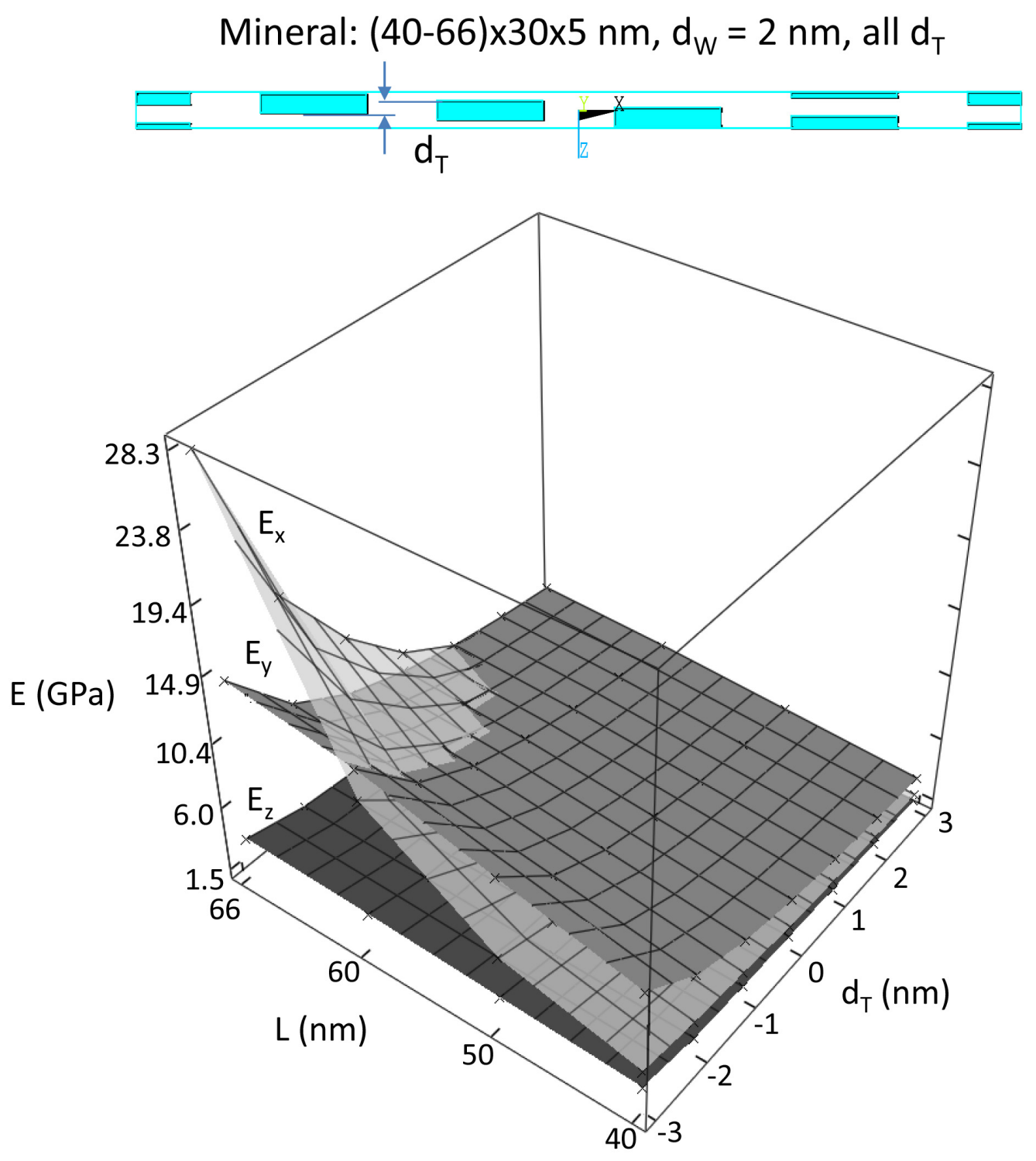

Figure 15: Variation of Young's moduli with the lateral distance $d_{T}$, in the range $[-3,3] \mathrm{nm}$, and the platelet length $L$ values $L=40,50,60,66 \mathrm{~nm}$ 

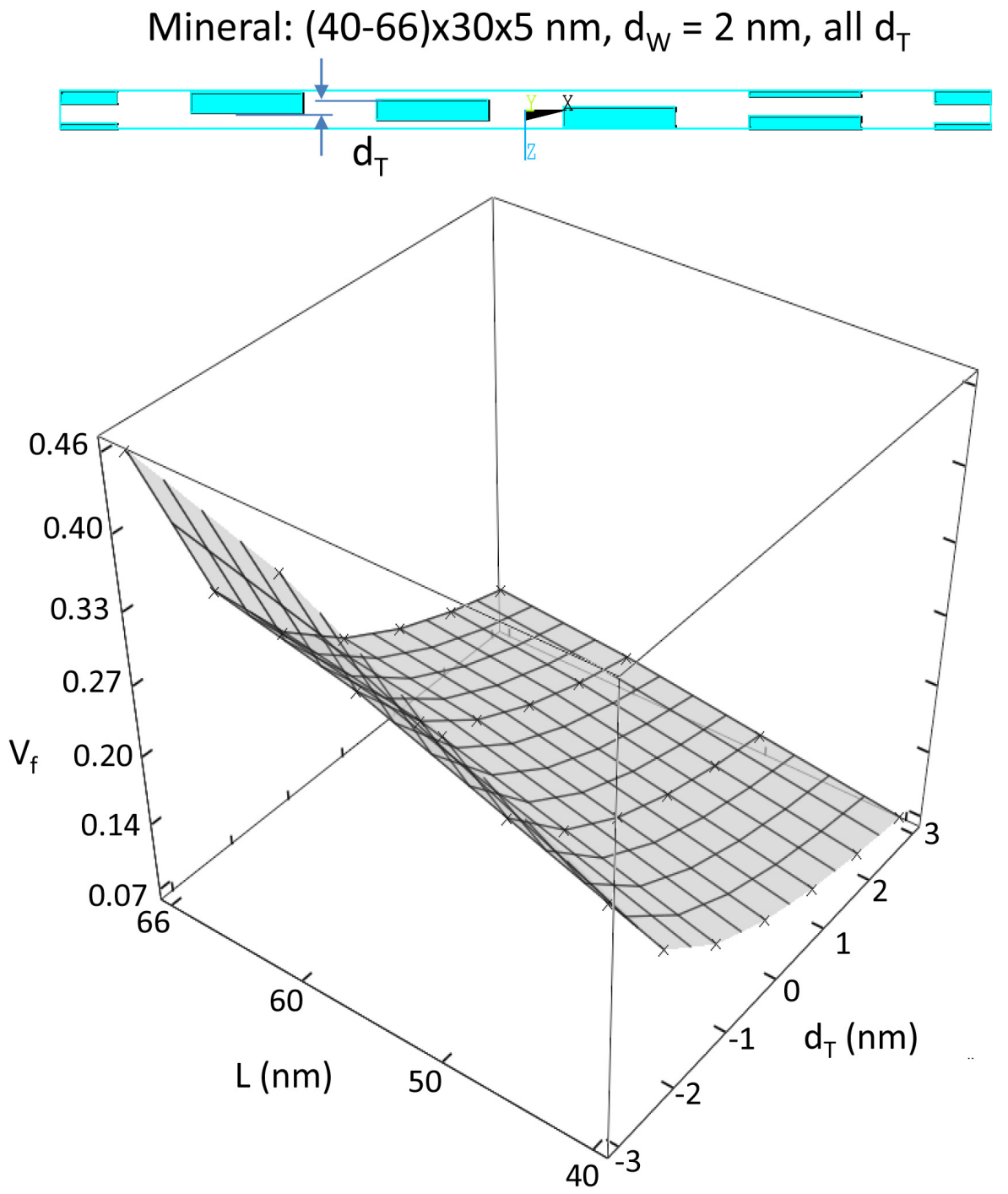

Figure 16: Variation of volume fraction of mineral $V_{f}$ with the lateral distance $d_{T}$, in the range $[-3,3] \mathrm{nm}$, and the platelet length $L$ values $L=40,50,60,66 \mathrm{~nm}$ 


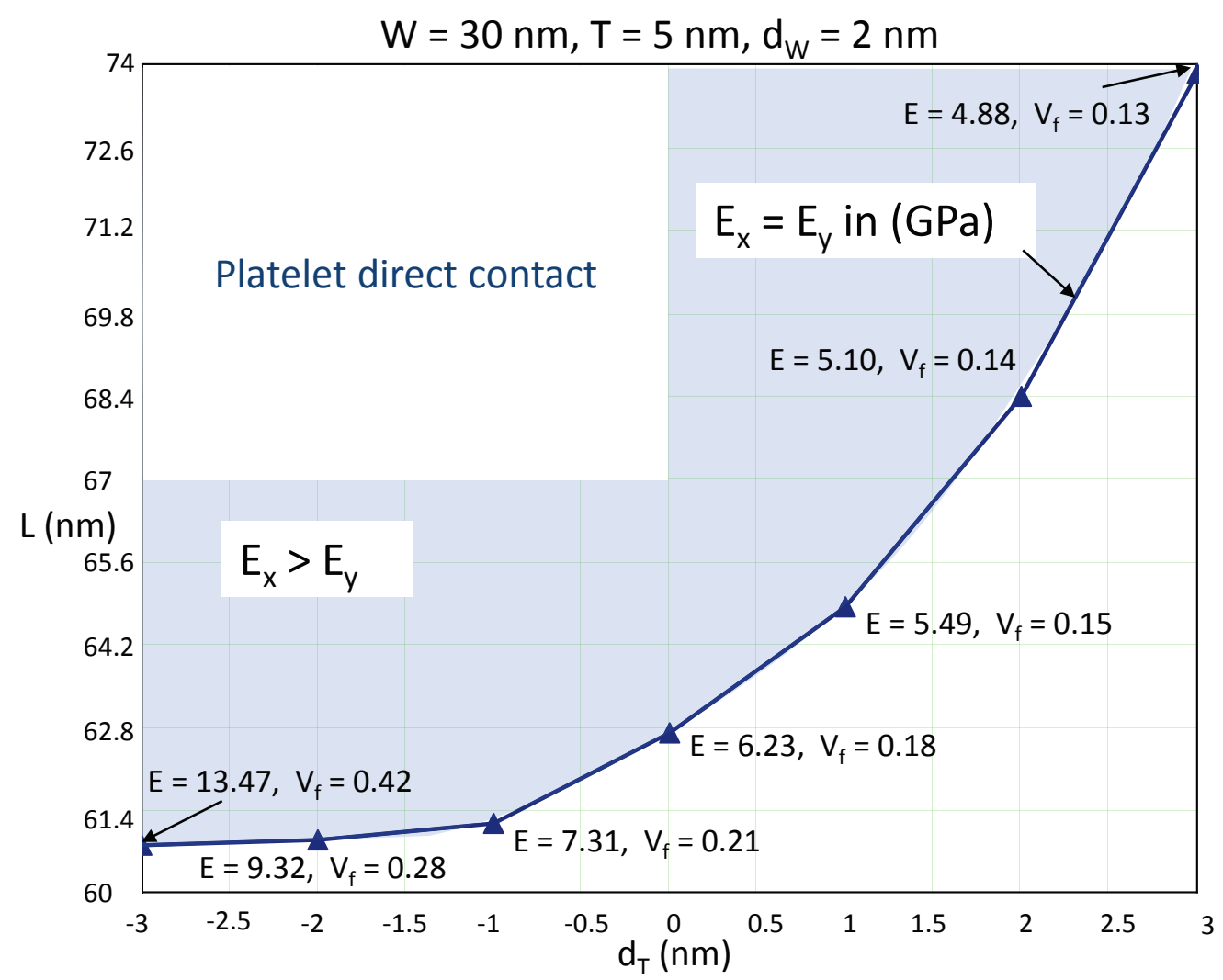

Figure 17: Platelet length $L$, for each lateral space $d_{T}$, that gives $E_{x}=E_{y}$ 


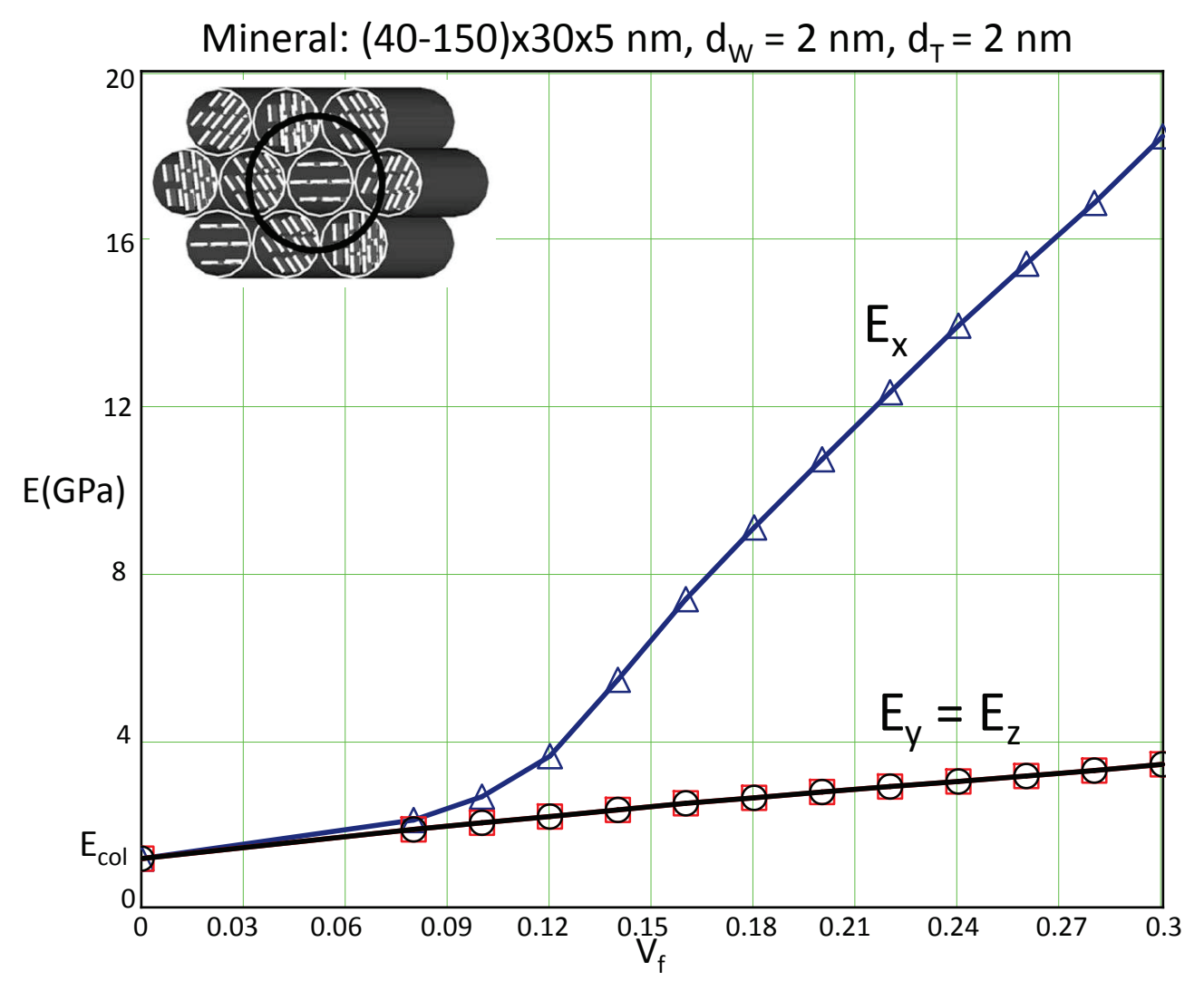

Figure 18: Variation of Young's moduli with the volume fraction of mineral for the fibril assembly within the fibre, by considering different platelet lengths in the range $L \in[40,150] \mathrm{nm}$. Inset: schematic representation of the fibril set reprinted from Rubin et al. (2003) with permission of Elevier 


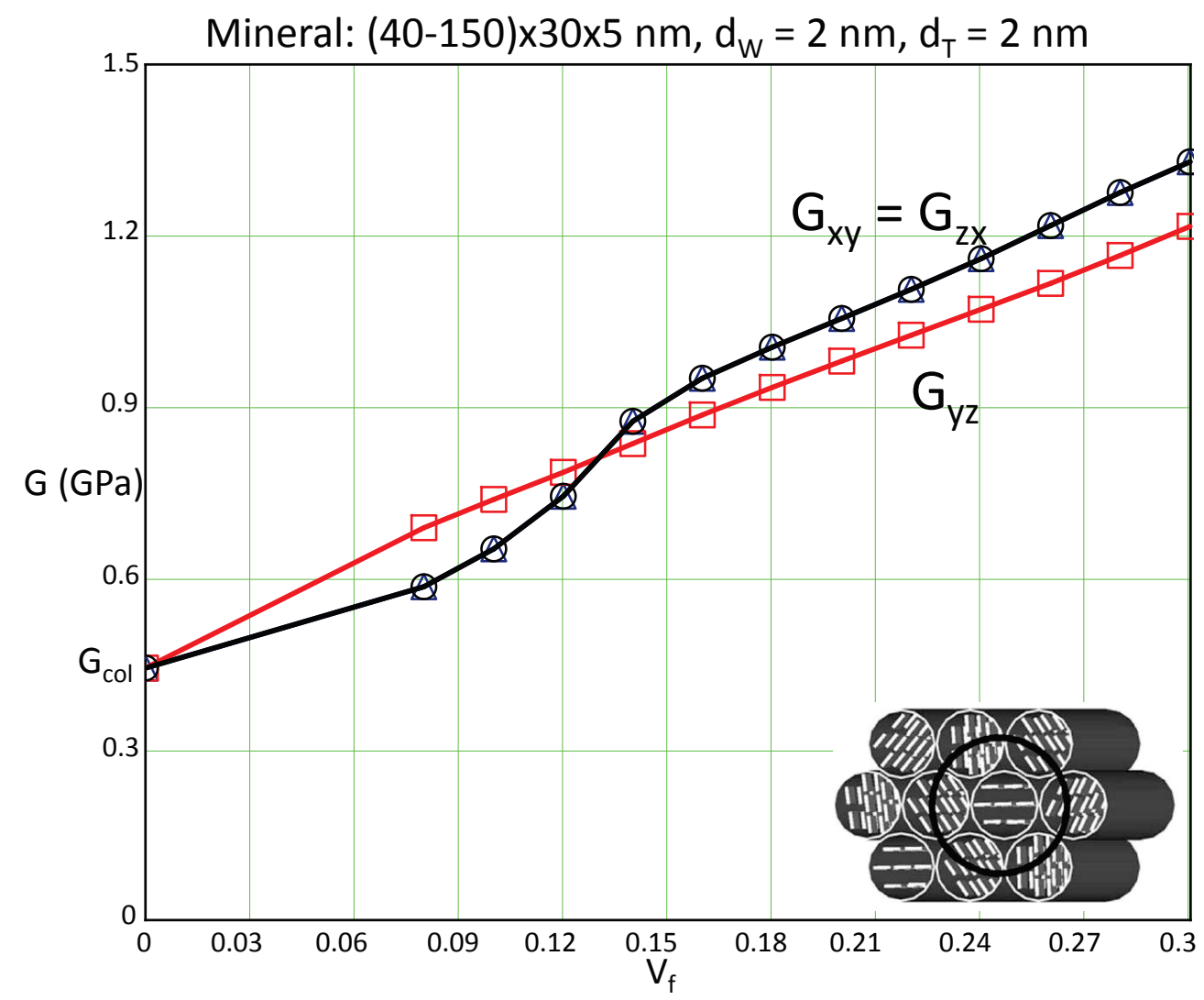

Figure 19: Variation of shear moduli with the volume fraction of mineral for the fibril assembly within the fibre, by considering different platelet lengths in the range $L \in$ [40,150] nm. Inset: schematic representation of the fibril set reprinted from Rubin et al. (2003) with permission of Elevier 


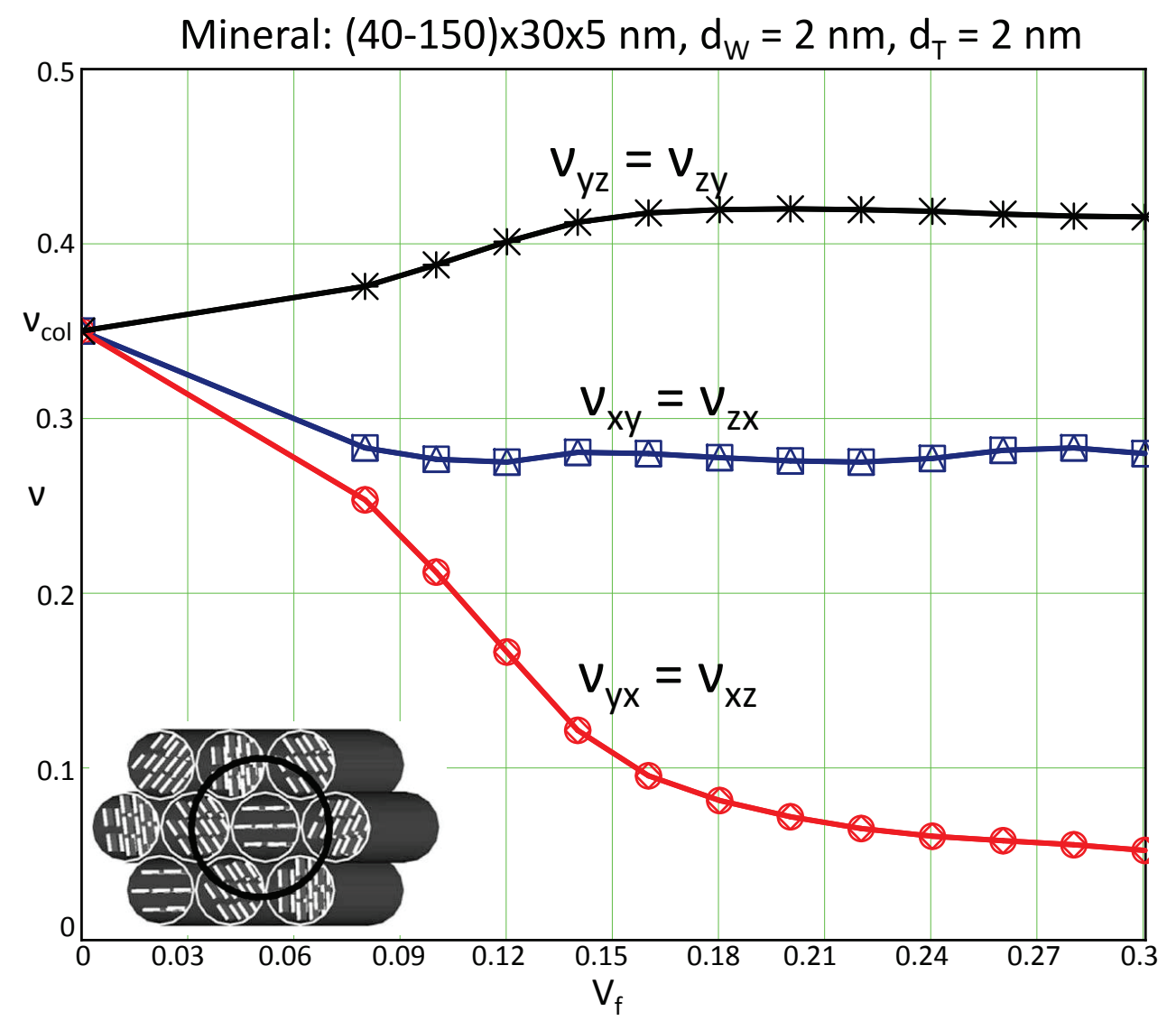

Figure 20: Poisson ratio variation with the volume fraction of mineral for the fibril assembly within the fibre, by considering different platelet lengths in the range $L \in[40,150] \mathrm{nm}$. Inset: schematic representation of the fibril set reprinted from Rubin et al. (2003) with permission of Elevier 


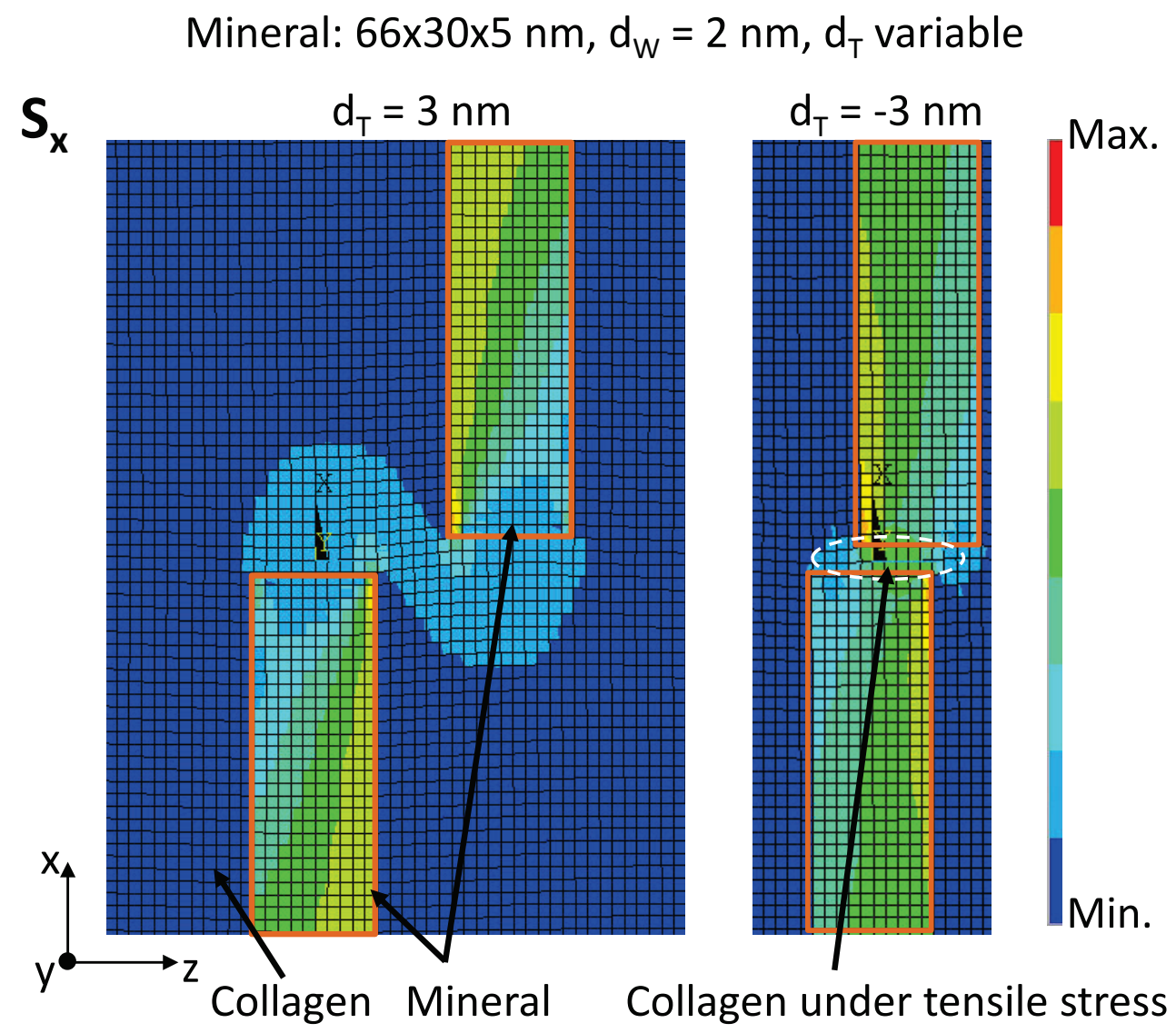

Figure 21: Detail of the element stress component $S_{x}$ when a uniform strain is applied in the $x$ direction. It has been considered a constant platelet length $L=66 \mathrm{~nm}$. Two different lateral spaces, $d_{T}$, between platelets are shown to analyze the load transfer through the collagen matrix: $d_{T}=3 \mathrm{~nm}$ on the left and $d_{T}=-3 \mathrm{~nm}$ on the right 


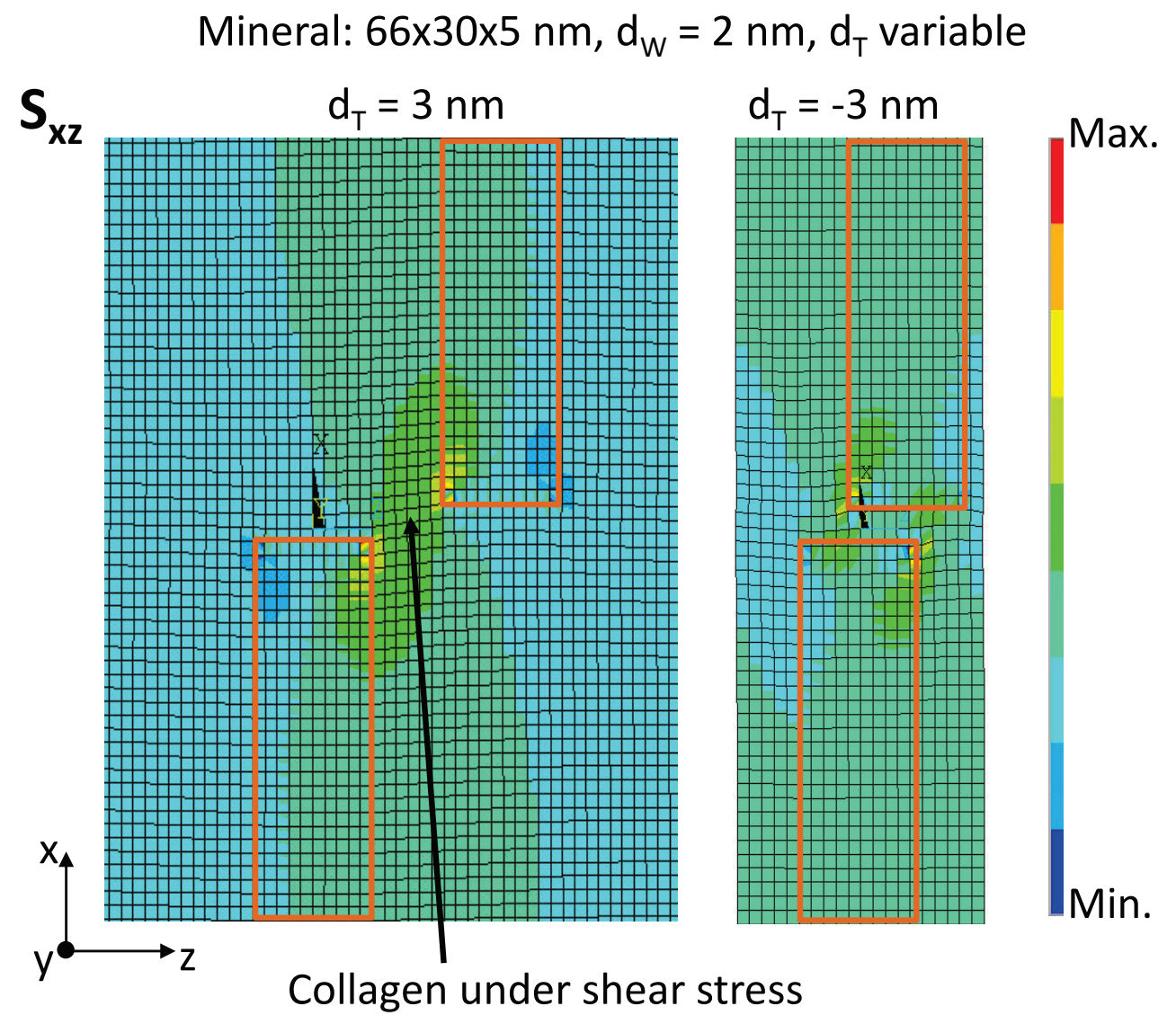

Figure 22: Detail of the element stress component $S_{x z}$ when a uniform strain is applied in the $x$ direction. It has been considered a constant platelet length $L=66 \mathrm{~nm}$. Two different lateral spaces, $d_{T}$, between platelets are shown to analyze the load transfer through the collagen matrix: $d_{T}=3 \mathrm{~nm}$ on the left and $d_{T}=-3 \mathrm{~nm}$ on the right 
Mineral: $L \times 30 \times 5 \mathrm{~nm}, \mathrm{~d}_{\mathrm{W}}=2 \mathrm{~nm}, \mathrm{~d}_{\mathrm{T}}=1 \mathrm{~nm}$
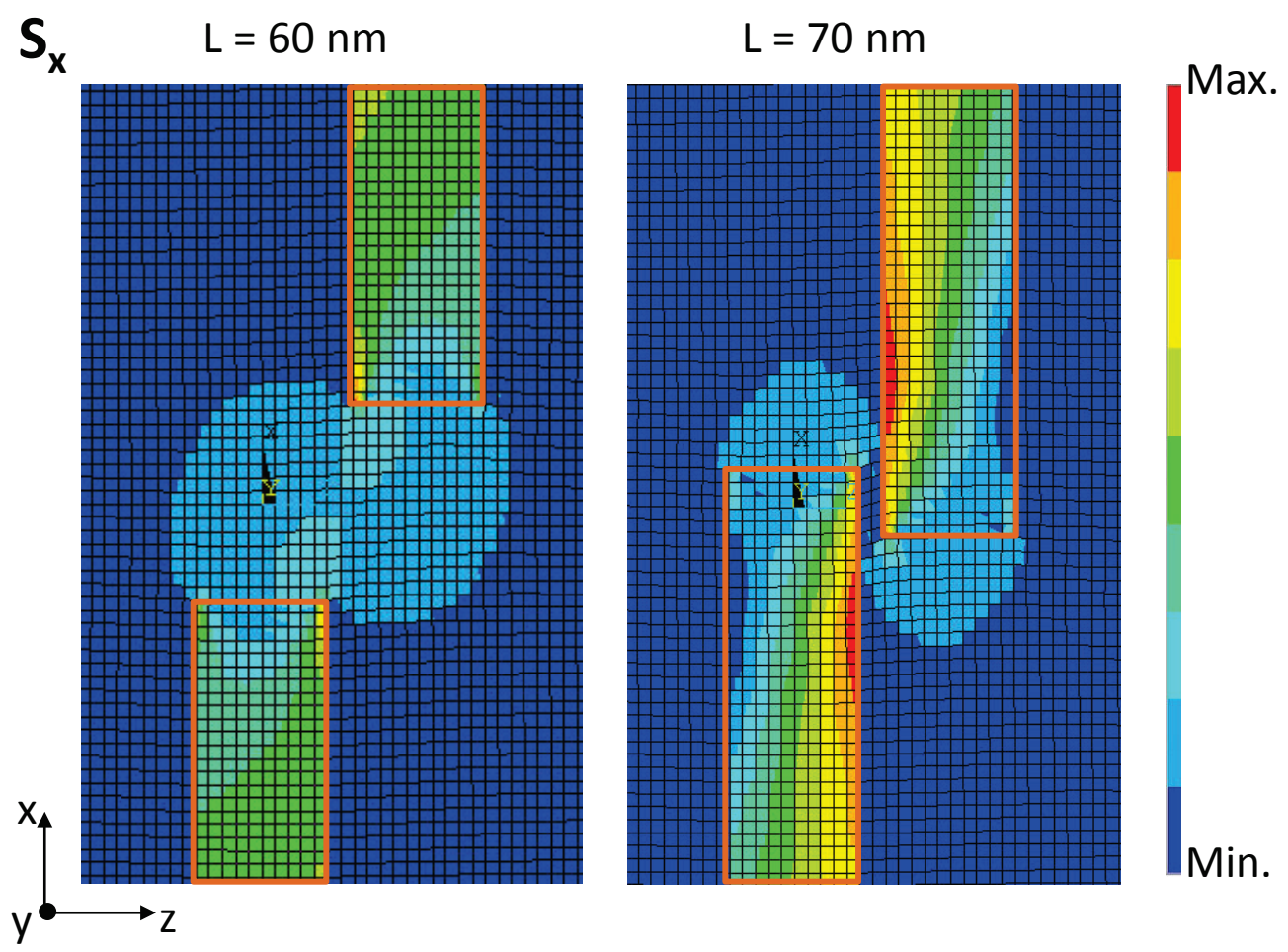

Figure 23: Detail of the element stress component $S_{x}$ when a uniform strain is applied in the $x$ direction. A constant lateral space between platelets $d_{T}=1 \mathrm{~nm}$ has been considered. Two platelet lengths are shown to analyze the load transfer through the collagen matrix: $L=60 \mathrm{~nm}$ on the left and $L=70 \mathrm{~nm}$ on the right 


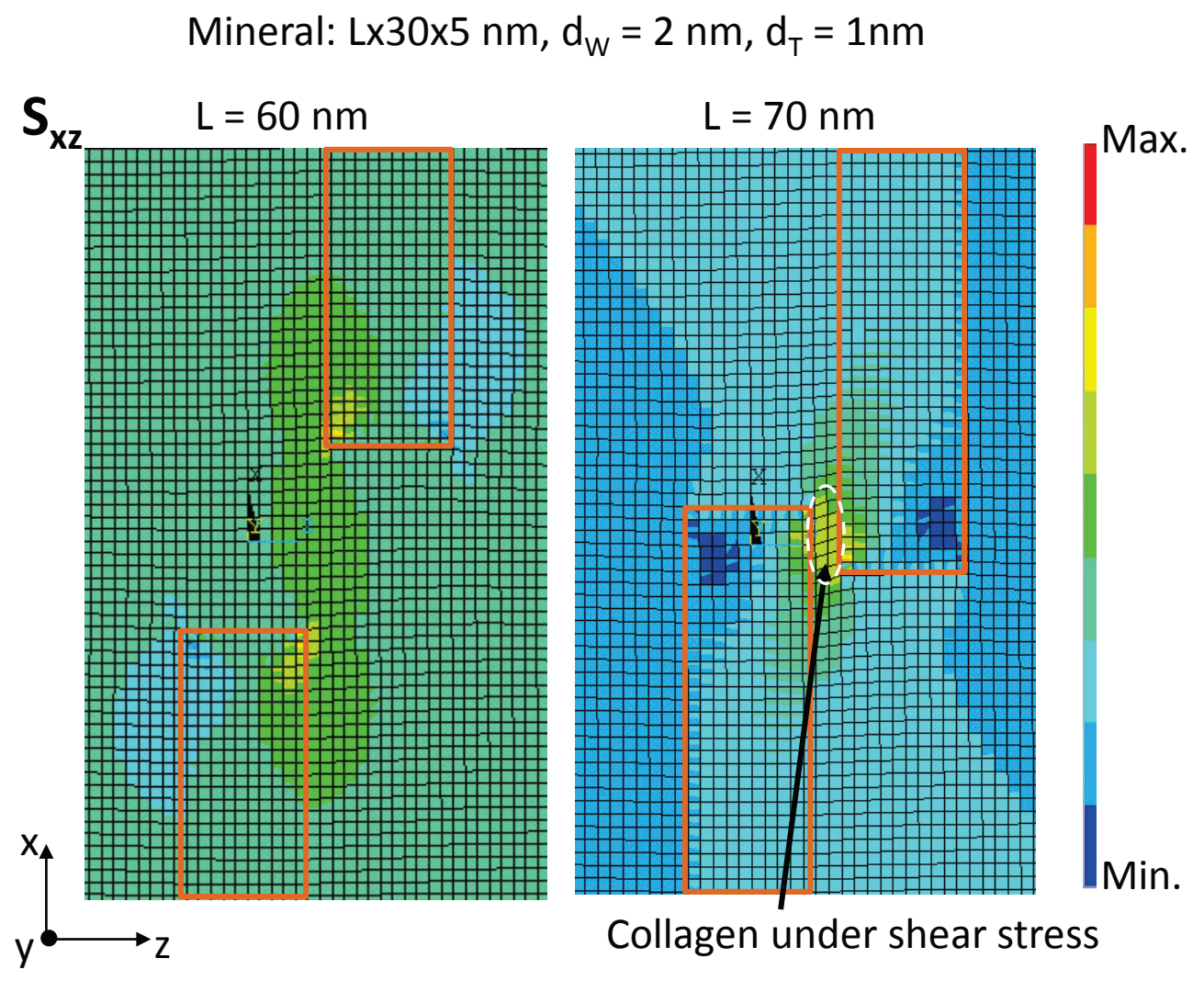

Figure 24: Detail of the element stress component $S_{x z}$ when a uniform strain is applied in the $x$ direction. A constant lateral space between platelets $d_{T}=1 \mathrm{~nm}$ has been considered. Two platelet lengths are shown to analyze the load transfer through the collagen matrix: $L=60 \mathrm{~nm}$ on the left and $L=70 \mathrm{~nm}$ on the right 


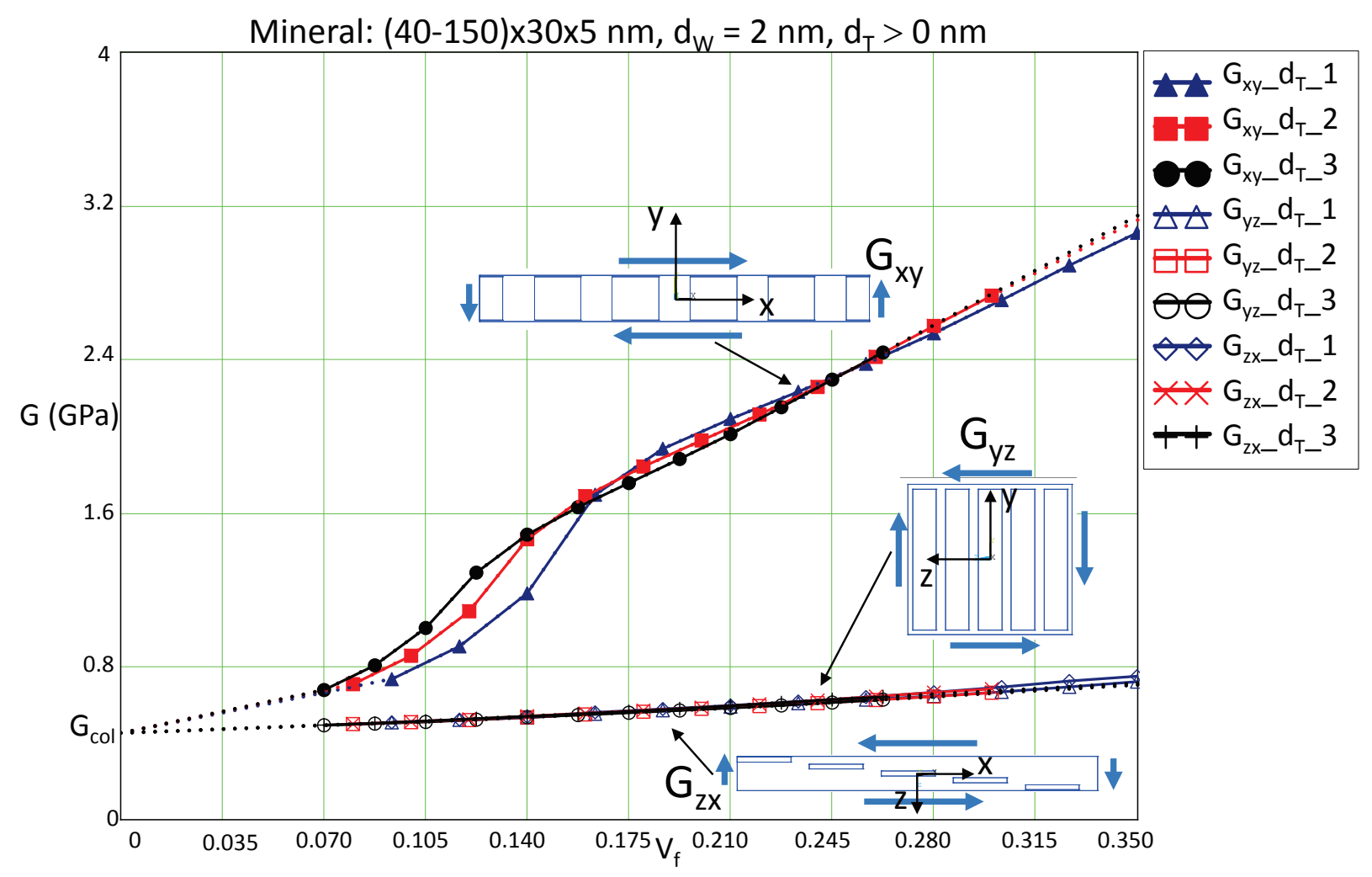

Figure 25: Variation of shear moduli with volume fraction of mineral by considering different platelet lengths, in the range $L \in[40,150] \mathrm{nm}$. Results are obtained for positive lateral space between platelets, $d_{T}=1,2,3 \mathrm{~nm}$ 


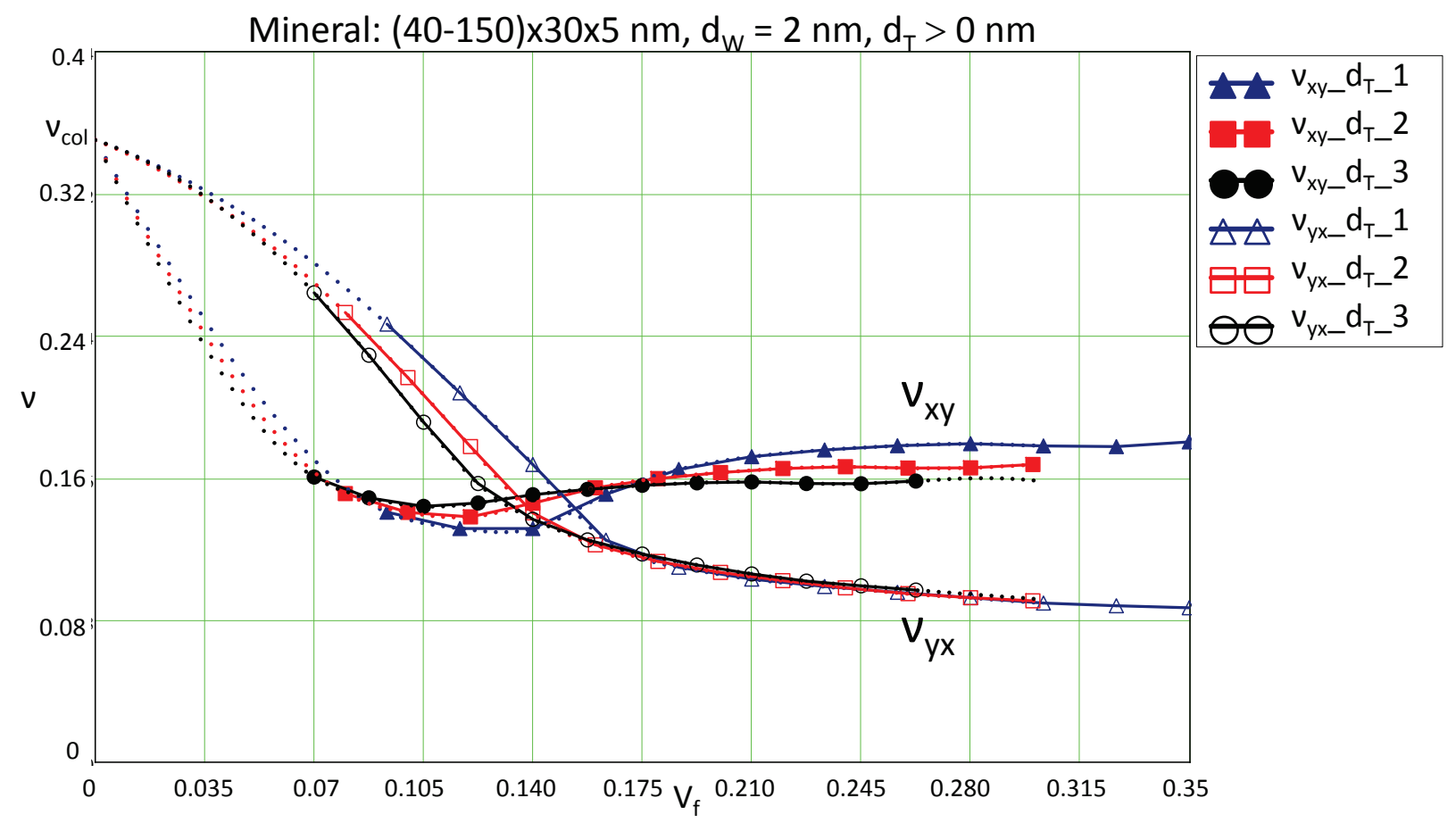

Figure 26: Variation of major and minor Poisson ratios $\nu_{x y}, \nu_{y x}$ with volume fraction of mineral by considering different platelet lengths, in the range $L \in[40,150] \mathrm{nm}$. Results are obtained for positive lateral space between platelets, $d_{T}=1,2,3 \mathrm{~nm}$ 


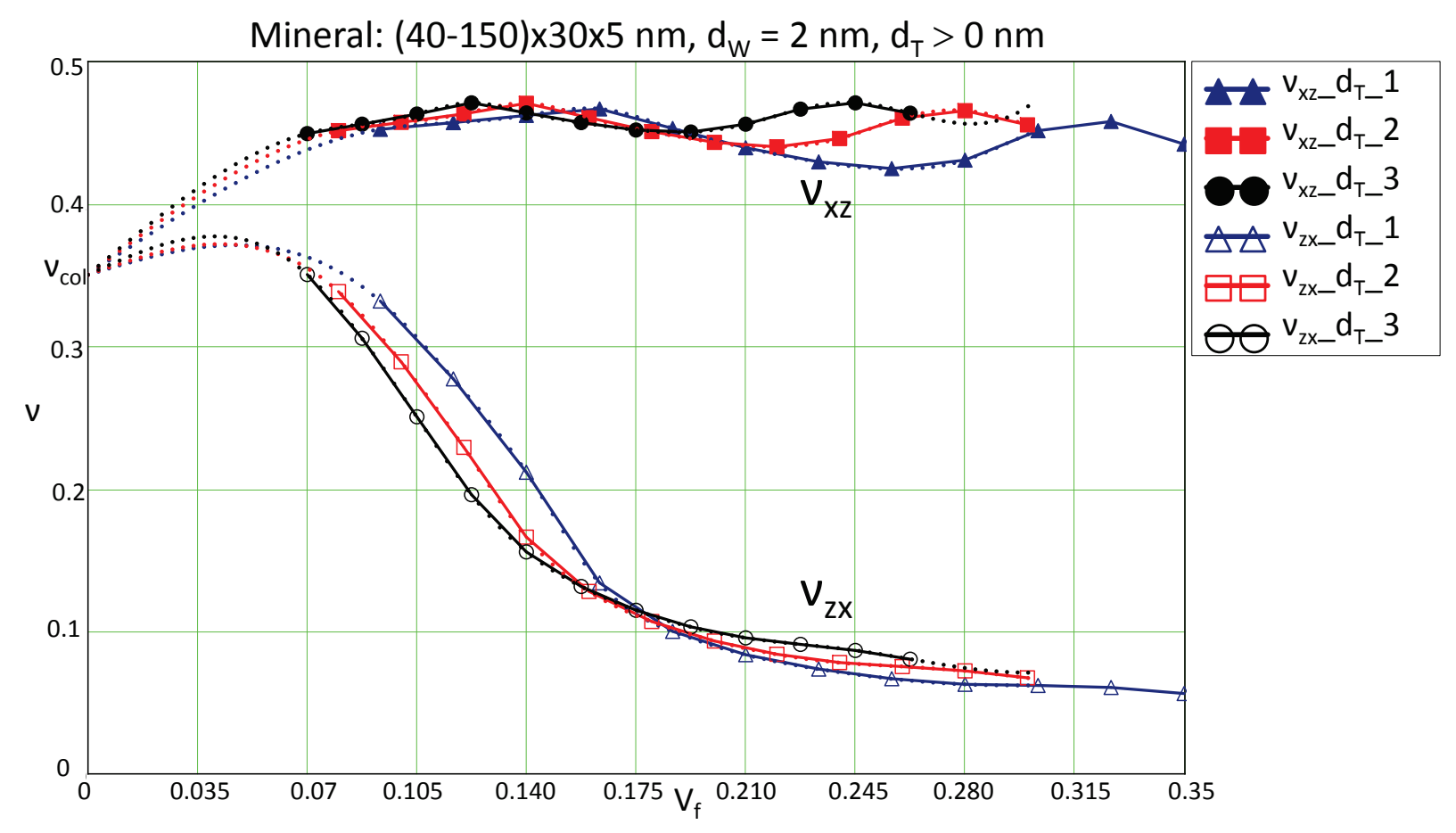

Figure 27: Variation of major and minor Poisson ratios $\nu_{x z}, \nu_{z x}$ with volume fraction of mineral by considering different platelet lengths, in the range $L \in[40,150] \mathrm{nm}$. Results are obtained for positive lateral space between platelets, $d_{T}=1,2,3 \mathrm{~nm}$ 


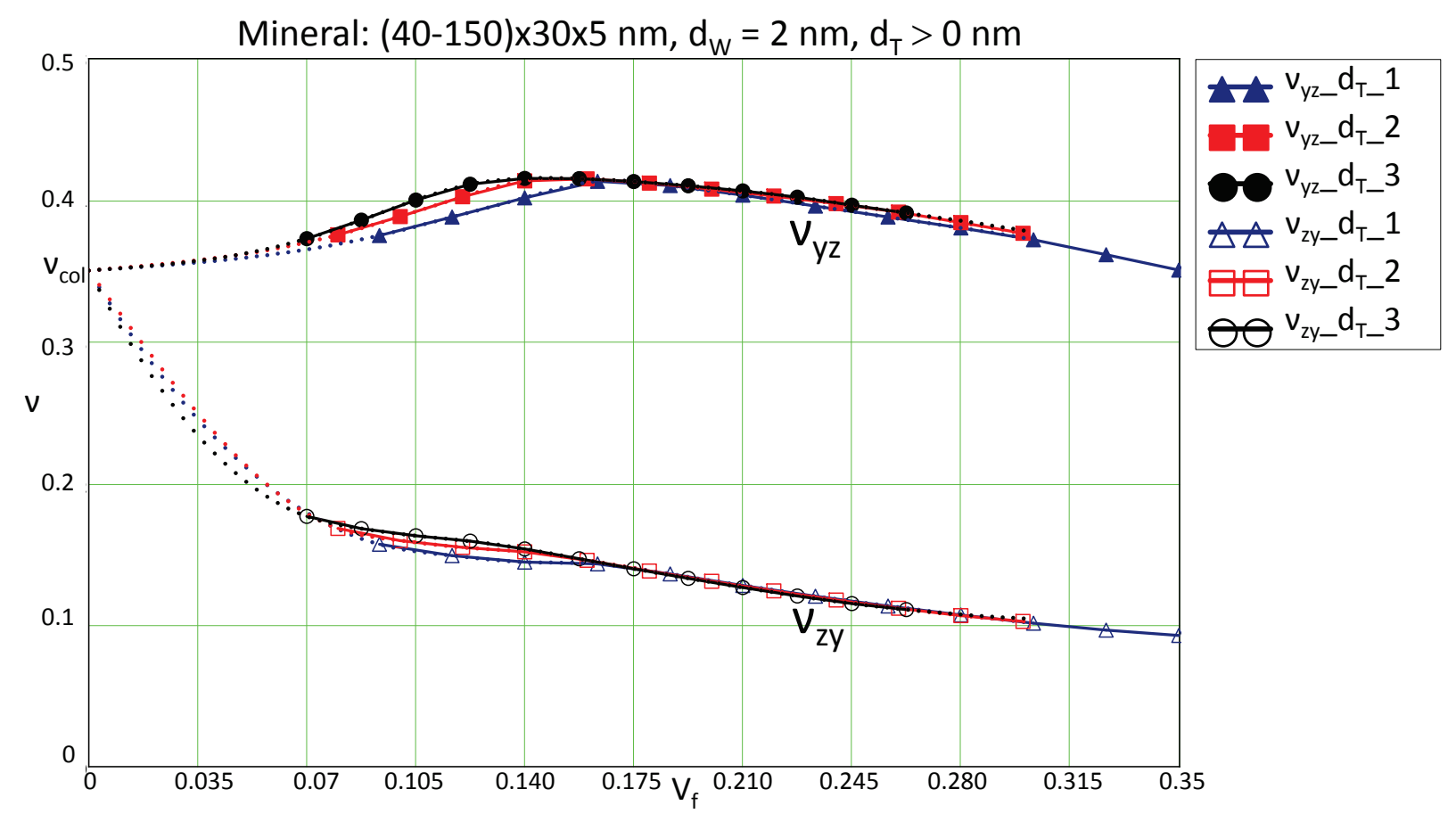

Figure 28: Variation of major and minor Poisson ratios $\nu_{y z}, \nu_{z y}$ with volume fraction of mineral. Results are obtained for positive lateral space between platelets, $d_{T}=1,2,3 \mathrm{~nm}$ and different platelet lengths, $L \in[40,150] \mathrm{nm}$ 